\title{
The effect of active grid initial conditions on high Reynolds number turbulence
}

\author{
R. J. Hearst · P. Lavoie
}

the date of receipt and acceptance should be inserted later

\begin{abstract}
The most expansive active grid parametric study to date is conducted in order to ascertain the relative importance of the various grid parameters. It is identified that the three most important parameters are the Rossby number, $R o$, the grid Reynolds number, $R e_{M}$, and the wing geometry. For $R o>50$, an asymptotic state in turbulence intensity is reached where increasing $R o$ further does not change the turbulence intensity while other parameters continue to vary. Three wing geometries are used: solid square wings, solid circular wings, and square wings with holes. It is shown that the wings with the greatest blockage produce the highest turbulence intensities and $R e_{\lambda}$, but that parameters such as the Kolmogorov, Taylor and integral scales are not significantly influenced by wing geometry. Finally, it is demonstrated that for several different sets of initial conditions that produce the same $R e_{\lambda}$, the spectra are collapsed everywhere but at the largest scales. This result suggests that regardless of the very different origins of the turbulence, the shape of the spectra at high wavenumbers is dependent only on $R e_{\lambda}$ when normalized by Kolmogorov variables, hence demonstrating a degree of independence from the initial conditions.
\end{abstract}

\section{Introduction}

Our traditional understanding of turbulence is derived from the Richardson-Kolmogorov cascade that describes energy being passed down from the large scales to successively smaller scales until it is dissipated as heat. According to this description, if the local Reynolds number, $R e_{\lambda}$, is sufficiently high, the turbulence can exhibit a universal state over a subset of

R. J. Hearst · P. Lavoie

Institute for Aerospace Studies, University of Toronto

Toronto, ON M3H 5T6, Canada

Tel.: +1-416-667-7716

Fax.: +1-416-667-7799

E-mail: lavoie@utias.utoronto.ca scales that are independent of the initial generating conditions. However, traditional grid turbulence experiments have demonstrated that there is a dependence of the produced turbulence on the initial conditions at low to moderate Reynolds numbers (Comte-Bellot and Corrsin, 1966; Lavoie et al., 2007). As such, the question of the relative importance of the initial conditions on the flow at high Reynolds numbers remains open.

Mydlarski and Warhaft (1996) constructed an active grid, based on the design of Makita (1991), to investigate high $R e_{\lambda}$ turbulence in an endeavour to approach the limit where the classical high $R e_{\lambda}$ physics may be observed. This style of active grid consists of a series of 'wings' mounted to rods that are actuated in a random pattern by stepper motors. The grid constructed by Mydlarski and Warhaft (1996) was an $8 M \times 8 M$ array, where $M$ is a mesh length, and it was placed in a $0.4 \mathrm{~m} \times 0.4 \mathrm{~m}$ wind tunnel. When comparing the turbulence produced by their grid to Kolmogorov's (1941) $k^{-5 / 3}$ law, they found that even $R e_{\lambda} \approx 500$ was not sufficient to satisfy high Reynolds number conditions. In a follow-up study, Mydlarski and Warhaft (1998) constructed a larger $8 M \times 8 M$ grid for a $0.9 \mathrm{~m} \times 0.9 \mathrm{~m}$ wind tunnel. Again, they found that for the increased $R e_{\lambda} \approx 730, k^{-5 / 3}$ behaviour was not reached for the velocity spectra, however, they did observe $k^{-5 / 3}$ scaling for the scalar (temperature) spectrum, suggesting that the scalar spectrum reaches a possible universal state earlier than the velocity fluctuations.

Since this seminal work by Mydlarski and Warhaft (1996, 1998), a number of other groups have designed active grids in an attempt to either reach high $R e_{\lambda}$ or to be able to control the characteristics of the generated turbulence. The most extensive parametric study of active grid generated turbulence to date was performed by Larssen and Devenport (2011) who placed more emphasis on the variability of the produced turbulence than on high $R e_{\lambda}$ turbulence theory. Larssen and Devenport (2011) placed a $10 M \times 10 M$ active grid in 
the contraction of a $1.83 \mathrm{~m} \times 1.83 \mathrm{~m}$ wind tunnel. By placing the grid in the contraction they were able to achieve global isotropy ratios near unity as originally demonstrated by Comte-Bellot and Corrsin (1966) with passive grids. In their study, Larssen and Devenport (2011) produced turbulence with $101 \leq R e_{\lambda} \leq 1362,2 \% \leq u^{\prime} / U \leq 12 \%$, and $0.24 \leq$ $L_{u x} / M \leq 3.19$, representing some of the highest $R e_{\lambda}$ and largest scale turbulence produced by a grid experiment to date. They found that the two parameters that were most important for characterizing the active grid-generated turbulence were the grid Reynolds number, $\operatorname{Re}_{M}=U M / v$, and the Rossby number, $R o=U / \Omega M$ (where $\Omega$ is the mean rotational velocity of the grid agitator wings).

A recent advancement by Bodenschatz et al. (2014) has introduced a 129 degree-of-freedom, $9 M \times 9 M$ active grid in a $1.5 \mathrm{~m} \times 1.5 \mathrm{~m}$ wind tunnel. Each wing of this grid is mechanically independent, unlike previous grids where all wings along a single axis were rigidly connected and moved together. With this new apparatus, Bewley et al. (2013) showed that with certain correlated motions of adjacent wings they were able to control the size of the large scale motion in the produced turbulence.

Several other Makita-style active grids have been designed and built for a variety of applications. Poorte and Biesheuvel (2002) used an active grid in water to investigate the motion of spherical bubbles in the grid wake. Kang et al. (2003) used an active grid to extend the work of ComteBellot and Corrsin (1971) to higher Reynolds numbers in order to compare the results with large eddy simulations. Cekli and van de Water (2010), Cal et al. (2010) and Knebel et al. (2011) have designed active grids to simulate atmospheric shear layers. Sytsma and Ukeiley (2013) investigated the effect of turbulence on lift generated by a flat plate, and Sharp et al. (2009) studied the impact of free-stream turbulence on a turbulent boundary layer. Finally, Thormann and Meneveau (2014) used fractal pattern wings to investigate how the turbulent kinetic energy decayed in the wake of such initial conditions.

The present study uses a novel variant of the Makita (1991) active grid to address questions concerning high $R e_{\lambda}$ physics and to gain a better understanding of the active grid parameter space. In particular, we produce high $R e_{\lambda}$ turbulence at a given $R e_{\lambda}$ with different initial conditions in order to assess if the turbulence shows any signs of 'remembering' how it was produced. The parametric study of Larssen and Devenport (2011) is also extended here in order to more fully understand the influence of active grid parameters on the produced turbulence.

The paper is organized as follows. Section 2 discusses the active grid design and experimental setup. Section 3 present the results of the parametric study identifying what grid parameters have the greatest influence over the produced turbulence. Section 4 then compares test cases from section 3 where similar $\operatorname{Re}_{\lambda}$ were produced with different initial conditions in order to assess if the turbulence spectra collapse for a given $R e_{\lambda}$, or if the differing initial conditions are able to produce turbulence with the same $R e_{\lambda}$, but different spectral shapes. Finally, section 5 presents the concluding arguments.

\section{Experimental Setup}

\subsection{Active grid design}

The active grid utilizes a double-mesh design of steel rods with diameter $6.35 \mathrm{~mm}$. The meshes are separated by $40 \mathrm{~mm}$ in the streamwise direction. Wings are mounted to the forward and aft meshes in an alternating pattern such that half the wings are on one mesh and the remaining wings are on the other, see Fig. 1. With this setup, adjacent wings are never in the same plane along a single rod. Therefore, the motion of adjacent wings can be decoupled, creating a more random spatial sequence than attainable by traditional active grids. The grid consists of 20 horizontal bars and 30 vertical bars, oriented as two layers of a $10 \times 15$ mesh, with a mesh length of $M=80 \mathrm{~mm}$. The only grid with greater variability is that of Bodenschatz et al. (2014), although the present grid has nearly twice the number of bars, which reduces the confinement effect of the tunnel size on the turbulence. Low friction cylindrical supports (diameter $12.7 \mathrm{~mm}$ ) hold the various meshes of the grid apart, and help maintain overall rigidity. While these supports were not required at every mesh length, they were placed at every junction in order to create a homogeneous background mesh. The 50 grid bars were actuated by Applied Motion Products STM23S-3RN integrated stepper motors. The motors were controlled via two RS-485 serial ports through a PC. Each motor was sent a top-hat distribution of: rotational velocity, $\Omega \pm \omega$; rotational period, $T \pm t$; and rotational acceleration, $A \pm \alpha$ (where in the formulation $B \pm \beta, B$ is the mean of the quantity and $\pm \beta$ represents the bounds of the random variation). The grid rods were rotated at rotational rates ranging from 0 to $20 \mathrm{~Hz}$. The acceleration was varied between 0 and $250 \mathrm{~Hz} / \mathrm{s}$. The average period of rotation was varied between 2 and 8 seconds. One of the limiting factors in the operation of the grid was that in order to prevent data collisions in the serial communication, a minimum time delay of $40 \mathrm{~ms}$ had to be enforced between communications to the grid.

Mounted to the rotating grid rods were 254 wings. Two wing geometries were tested: square wings $(55 \mathrm{~mm} \times 55 \mathrm{~mm})$, and circular wings (diameter $55 \mathrm{~mm}$ ). Holes of diameter $20 \mathrm{~mm}$ were cut out of the square wings, and tests were conducted both with the holes open and with them covered by aluminum tape (as depicted in Fig. 1(b)). Circular wings have not previously been investigated in the literature and were introduced here to remove the sharp corners of the square 
wings with the intention of potentially avoiding streamwise vortices from the edges that could induce long streamwise correlations. Schematics of the wing geometries are shown in Fig. 2.

The active grid was operated in one of 8 modes. In fully random (FR) mode, every rod received a unique random signal, and there was no deliberate correlation between the motion of the grid rods. In classical (CL) mode, rod pairs mounted immediately upstream/downstream of one another on the forward and aft meshes were deliberately sent the same signal so that the grid behaved as if there was only one mesh, simulating a traditional active grid. These two base modes, FR and CL, were also used to create a series of grid control sequences with varying degrees of correlation between grid rods. For instance, in 2FR mode, two adjacent rods on one of the meshes received the same signal. The CL mode that corresponds to $2 \mathrm{FR}$ is $4 \mathrm{CL}$, where two adjacent rods on the forward mesh received the same signal, and the two rods on the aft mesh immediately downstream of the first two rods also received that the same signal, i.e., a total of 4 rods received the same signal and moved in unison. All operational modes used in this study are summarized in Table 1.

\subsection{Instrumentation}

All experiments were performed in the low-speed, recirculating wind tunnel at the University of Toronto Institute for Aerospace Studies. The wind tunnel has a hexagonal testsection that is $1.2 \mathrm{~m}$ wide, $0.8 \mathrm{~m}$ tall and $5 \mathrm{~m}$ long. The hexagonal corners are adjustable so that an approximately zero pressure gradient may be achieved along the test-section length Tests were conducted with mean velocities between $3 \mathrm{~m} / \mathrm{s}$ and $16 \mathrm{~m} / \mathrm{s}$. The background turbulence intensity in the wind tunnel does not exceed $0.06 \%$ in this velocity range.

Measurements were performed with a constant temperature hot-wire anemometer built at the University of Newcastle (Miller et al., 1987). Both single-wires and X-wires were employed. Single-wires (SW1, SW2, and SW3) with different sensing lengths were used in order to verify that the results were not a consequence of limited spatial resolution. Wire dimensions are given in Table 2, where $\ell$ is the sensing length and $d$ is the wire diameter. The single-wires were made in-house with tungsten wire. The region outside of the sensing length was coated with copper. The tolerance on wire sensing length was $\pm 0.1 \mathrm{~mm}$. Two X-wires (XW1 and XW2) with different sensing lengths were used. XW1 was an Auspex A55P51 probe. XW2 was made in-house on the same prongs as $\mathrm{XW} 1$, but with smaller wire. The mean Kolmogorov microscale $\left(\eta=v^{3 / 4} /\langle\epsilon\rangle^{1 / 4}\right)$ over all measurements was $\sim 0.2 \mathrm{~mm}$, and typical probe resolutions are provided in Table 2 based on this value. The hot-wires were
Table 2: Hot-wire probe dimensions.

\begin{tabular}{c|ccccc}
\hline & SW1, SW2 & SW3 & XW1 & XW2 & \\
\hline \hline$\ell$ & 1.1 & 0.6 & 1.2 & 0.6 & $\mathrm{~mm}$ \\
$d$ & 5.0 & 2.5 & 5.0 & 2.5 & $\mu \mathrm{m}$ \\
$\ell / \eta$ & 5.5 & 3.0 & 6.0 & 3.0 & \\
\hline
\end{tabular}

operated with an overheat ratio of 1.6 and an analog cutoff filter at $f_{c}=9.2 \mathrm{kHz}$. The sampling frequency was set to $f_{s}=2 f_{c}+1 \mathrm{kHz}$. Data were acquired with a 16-bit National Instruments PCI-6259 card. Samples were taken for 4 minutes or longer to ensure $\pm 1 \%$ statistical convergence of $\left\langle q^{2}\right\rangle=\left\langle u^{2}\right\rangle+2\left\langle v^{2}\right\rangle$ using the $95 \%$ confidence interval (Benedict and Gould, 1996); it was verified that $\left\langle v^{2}\right\rangle \approx\left\langle w^{2}\right\rangle$ in the present flow by rotating the $\mathrm{X}$-wire $90^{\circ}$. Single-wires were calibrated with a $4^{\text {th }}$-order polynomial fit to 10 velocities. The X-wire was calibrated over 10 velocities and 7 angles using the look-up table approach discussed by Burattini and Antonia (2005). All calibrations were performed in situ with the grid set to its fully open position (15\% blockage with $1.7 \%$ turbulence intensity at $x / M=30$ ).

The principal setup employed SW1 and XW1 separated by $10 \mathrm{~mm}$, and mounted to a 4 degree-of-freedom ( 3 translational and 1 rotational) traverse system. The home position of the system was at the centre of the tunnel cross-section. This setup was used to perform both parametric study measurements at the centre of the tunnel cross-section and to perform transverse planar measurements for the assessment of flow homogeneity. The secondary setup featured two single-wires, SW2 mounted to a fixed stand at $y / H=+0.14$ ( $H=400 \mathrm{~mm}$ is the tunnel half-height), and SW1 mounted to a vertical traverse system. These probes were sequentially separated by known amounts in order to determine the transverse correlation, $B_{u u}\left(r_{y}\right)=\left\langle u(x) u\left(x+r_{y}\right)\right\rangle$, at various separations, $r_{y}$, along the $y$-axis. This correlation was then used to compute the transverse integral length scale, $L_{u y}$, from

$L_{\alpha \beta}=\frac{1}{\left\langle\alpha^{2}\right\rangle} \int_{0}^{r_{\beta, 0}} B_{\alpha \alpha}\left(r_{\beta}\right) \mathrm{d} r_{\beta}$,

where $\alpha=u, v$, or $w, \beta=x, y$, or $z$ and $r_{\beta, 0}$ is the first zerocrossing of the autocorrelation $B_{\alpha \alpha}\left(r_{\beta}\right)=\left\langle\alpha(x) \alpha\left(x+r_{\beta}\right)\right\rangle$ (ComteBellot and Corrsin, 1971). The majority of the parametric study measurements and all $L_{u y}$ measurements were conducted at $x / M=30$, with some other measurements investigating the effect of $\operatorname{Re}_{M}$ on constant grid conditions being conducted with SW3 and XW2 at $x / M=41$. The significance of these measurements being conducted with different resolution and at a different location is discussed in section 4.

Measurements of the pressure drop across the grid were performed with a 10 Torr MKS pressure transducer con- 
Table 1: Description of grid operational modes.

\begin{tabular}{l||cccccccc}
\hline Mode & FR & CL & 2FR & 3FR & 4CL & 5FR & 6CL & 10CL \\
\hline \hline Front \& aft mesh synchronized & No & Yes & No & No & Yes & No & Yes & Yes \\
\hline Number of bars receiving same signal & 0 & 2 & 2 & 3 & 4 & 5 & 6 & 10 \\
\hline Number of independent signals to grid & 50 & 25 & 24 & 18 & 12 & 10 & 9 & 5 \\
\hline
\end{tabular}

nected to static pressure ports upstream and downstream of the active grid. The pressure was sampled simultaneously with the velocity measurements, and thus was acquired over 4 minutes.

\subsection{Estimation of turbulent quantities}

The turbulent quantities used throughout this study are briefly defined here. Unless stated otherwise, the turbulence intensity was calculated based on $\left\langle q^{2}\right\rangle$, where $\langle\cdot\rangle$ denotes a timeaverage. For convenience, we define the parameter $T_{q}$ as

$T_{q}=\frac{\left\langle q^{2}\right\rangle^{1 / 2}}{3^{1 / 2} U}$

as the total turbulence intensity. The global isotropy was evaluated from the ratio $u^{\prime} / v^{\prime}=\left\langle u^{2}\right\rangle^{1 / 2} /\left\langle v^{2}\right\rangle^{1 / 2}$. The mean turbulent kinetic energy dissipation rate was estimated from

$\langle\epsilon\rangle=3 v\left[\left\langle\left(\frac{\partial u}{\partial x}\right)^{2}\right\rangle+2\left\langle\left(\frac{\partial v}{\partial x}\right)^{2}\right\rangle\right]$

which is based on the less restrictive assumption of local homogeneity rather than local isotropy. The Taylor microscale was similarly calculated allowing for variations from local isotropy with,

$\lambda^{2}=5 v \frac{\left\langle q^{2}\right\rangle}{\langle\epsilon\rangle}$

and in turn the Taylor microscale Reynolds number was estimated from

$\operatorname{Re}_{\lambda}=\frac{\left\langle q^{2}\right\rangle^{1 / 2} \lambda}{3^{1 / 2} v}$

The longitudinal integral scale, $L_{u x}$, was estimated using (1) and the autocorrelation based on the time-series of the $u$ measurements. Finally, the normalised pressure drop across the grid is given by,

$C_{P}=\frac{\Delta P}{\frac{1}{2} \rho U^{2}}$,

where $\Delta P$ is the measured pressure drop.

\subsection{Post-processing and uncertainty estimates}

Post-processing was performed in order to improve the estimates of the statistical turbulent quantities. The successive filtering technique of Mi et al. $(2005,2011)$ was used to filter the data at the Kolmogorov frequency, $f_{K}=U /(2 \pi \eta)$ in order to eliminate high-frequency noise. This technique involves estimating $f_{K}$, then applying a digital low-pass filter at $f_{K}$ and updating the estimate of $f_{K}$ until a converged value is reached. In the present analysis, a $9^{\text {th }}$-order Butterworth filter was used to digitally filter at $f_{K}$. Convergence was typically reached in 4 or fewer iterations. Wyngaardstyle corrections were made to the variances of the velocity fluctuations and velocity derivatives in order to minimize the error due to finite spatial resolution (Wyngaard, 1968; Zhu and Antonia, 1996; Hearst et al., 2012). Typical corrections for the velocity variances are $\pm 0.5 \%$, and corrections for the gradients may reach $\pm 10 \%$ for the smallest $\eta$ cases. A more detailed description of the post-processing used here is given by Hearst and Lavoie (2014).

Estimates of the measurement uncertainties are provided throughout this work as 'error bars' on the figures. In general, we only show a few error bars per figure to provide a visual representation of the uncertainty while keeping clutter low. If an error bar is not visible on an entire plot, then the uncertainty is contained within the symbol size. Uncertainties were estimated as the quadrature addition of the bias and random uncertainties. Random uncertainties were estimated using the boot-strapping technique described by Benedict and Gould (1996), and bias uncertainties were estimated from the break-down provided by Jørgensen (2002). Although the uncertainty changes marginally for each measurement, they are generally within the following bands: $\pm 1 \%$ for $U, \pm 2.5 \%$ for $\left\langle u^{2}\right\rangle, \pm 3 \%$ for $\left\langle v^{2}\right\rangle, \pm 5 \%$ for $\left\langle(\partial u / \partial x)^{2}\right\rangle$, and $\pm 6 \%$ for $\left\langle(\partial v / \partial x)^{2}\right\rangle$. The uncertainty on more complex quantities was calculated based on standard error propagation rules and quadrature addition.

\section{Influence of active grid parameters on the produced turbulence}

The effect of the various adjustable parameters of the active grid are investigated here to determine their influence 
on the produced turbulence. The present parametric study extends that conducted by Larssen and Devenport (2011), as the present apparatus has more adjustable parameters that can be varied over a greater range. In particular, we take many of the grid parameters to asymptotes not observed in previous studies, thus identifying where their influence becomes limited. As will be shown, it highlights the possibility of controlling certain features of the turbulence while allowing others to remain unchanged.

In this section, we first investigate the homogeneity of the flow field produced by the grid. We then briefly touch on parameters that do not have a significant influence on the flow, before individually addressing those that exert significant authority over the produced turbulence. Detailed results from this parametric study are tabulated in Appendix A. Any test referred to by name can be found there. Test names ending with an 'a' are conducted with square wings with holes, with a 'b' are conducted with solid square wings, and with a 'c' are conducted with solid circular wings.

\subsection{Homogeneity}

The homogeneity of the flow is assessed in Fig. 3. Two FR mode (V4a and V6a) and a 10CL mode (C13a) test case are considered. These cases were chosen because they are representative of the parameter space. In particular, C13a represents the 'worst-case' as it is the most correlated sequence, and V6a represents a high rotational rate fully random case. While the homogeneity of the streamwise turbulence intensity and the global isotropy fall within the uncertainty bounds for these two quantities for all three test cases, the mean velocity changes by nearly $3 \%$ within the investigation range for case $\mathrm{C} 13 \mathrm{a}$. The two FR mode cases have homogeneous $U$ to within $\pm 0.5 \%$. The 10CL mode operation may effectively cause a shear region near the centreline of the tunnel because all the wings above the centreline receive the same signal, and all the wings below the centreline receive a different signal. The value of the correlation coefficient, $\langle u v\rangle / u^{\prime} v^{\prime}$ shown here is within the range measured in the far-field of regular grids, e.g., Isaza et al. (2014), for all three test cases. The two FR mode test cases shown have rotational rates of $\Omega \pm \omega=3 \pm 2 \mathrm{~Hz}$ (V4a) and $\Omega \pm \omega=8 \pm 7 \mathrm{~Hz}$ (V6a), thus identifying that the homogeneity is a consequence of the operational mode rather than $\Omega$. The homogeneity of FR mode is verified over a larger range for V6a in Fig. 4, where the $z / M=0$ homogeneity scan axis is plotted with the vertical scan from the transverse integral measurements, showing the homogeneity extends over at least the centre $50 \%$ of the tunnel height. While only the central region of the wind tunnel is assessed here, several other active grids have demonstrated that active grid turbulence is typically homogeneous when operated in a random mode unless measurements are performed in close proximity to the walls (Makita, 1991; Mydlarski and Warhaft, 1996; Poorte and Biesheuvel, 2002; Larssen and Devenport, 2011).

The only effect of correlating the motion of adjacent wings for this apparatus appears to be adversely changing the homogeneity. FR mode is thus used for all test cases, except those specifically investigating the influence of different correlated sequences, since FR mode produces the most homogeneous flow.

\subsection{Parameters that do not affect the produced turbulence}

The wing rotational period $(T \pm t)$, i.e., the amount of time a wing moves in a given direction at a given rate before it receives a new signal, does not measurably influence the produced turbulence, as indicated by tests T1a-T6a in Table A2. As such, all other tests were conducted with $T \pm t \approx$ $2.1 \pm 2.0$ seconds, unless stated otherwise.

The rotational acceleration $(A \pm \alpha)$ was also found to not significantly influence the produced turbulence. The particular tests designed to investigate the acceleration were A1a-A8a in Table A2. In these tests, the majority of the turbulence parameters were invariant with $A$ within the uncertainty bounds of the experiment. Tests A1a and A3a do exhibit large values of $L_{u x}$ relative to the other measurements, but a coherent trend is not discernible, suggesting those points are likely outliers. In further support of this hypothesis, the other streamwise length scale, $L_{v x}$, was constant to within $\pm 5 \%$ and the uncertainty of our ability to estimate it for cases A1a-A8a. The present finding contrasts with the results of Larssen and Devenport (2011) who found that the turbulence intensity dropped by $0.6 \%$ (absolute) over the acceleration range $5 \mathrm{~Hz} / \mathrm{s}$ to $20 \mathrm{~Hz} / \mathrm{s}$. In the present study, rotational accelerations in the range $0.5 \mathrm{~Hz} / \mathrm{s} \leq A \pm \alpha \leq 250 \mathrm{~Hz} / \mathrm{s}$ were investigated, significantly extending the investigation region for this parameter, and it was found that the previously observed trend may be absorbed into the scatter of the present measurements, revealing the produced turbulence was not affected in a systematic way by rotational acceleration.

The difference between FR and CL modes was rigorously assessed through test pairs V3a and S5a, V4a and S6a, and T1a and S7a. In general, it was found that there was no marked difference between operating the grid in FR mode or CL mode. The influence of the more correlated sequences was assessed through tests C1a-C13a. While Fig. 3 demonstrates that the correlated sequences produce a less homogeneous flow field (with respect to $U$ only), these sequences have little to no effect on the other turbulence statistics. This result differs from that of Bewley et al. (2013) who were able to control the autocorrelation functions of the velocity components by correlating the motion of grid elements. We 
attribute this discrepancy to their ability to control the rotation of pairs and tetrads of adjacent wings, thus creating a strong localized correlation in the initial generating conditions. Such correlation is not possible in the present grid because multiple wings are still mounted to a single rod.

The influence of the choice of random distribution function was investigated by tests G1a-G4a. For these tests, a Gaussian distribution was used rather than a top-hat. In general, it was found that the influence of the random distribution was not significant, and as such, all other tests were conducted with a top-hat distribution.

Finally, tests R1a-R3a assess the impact of forcing the rotation direction to change with each new command. When compared to test cases where this was not enforced, it was found that there was no systematic influence of applying this protocol.

\subsection{Influence of the mean wing rotational rate}

This section focusses on the influence of the wing rotational rate when the global Reynolds number is held constant at $R e_{M} \approx 39,000$. The instantaneous wing rotational rate was varied between $0.25 \mathrm{~Hz}$ and $20 \mathrm{~Hz}$, and results are plotted in Fig. 5 versus the Rossby number $(R o=U / \Omega M)$. Fig. 5(a) shows that for $U / \Omega M>50$ the turbulence reached a constant state whereby decreasing the rotational rate further did not change the turbulence intensity. However, for high values of $\Omega$ and Rossby numbers in the range $U / \Omega M<50$, there was significant variability in the turbulence intensity. The $U / \Omega M=50$ limit was not as well defined for $R e_{\lambda}$ as demonstrated in Fig. 5(b). This is due to the dependence of $R e_{\lambda}$ on $\lambda$, which also does not plateau as abruptly as the turbulence intensity (Fig. 5(e)). While the dissipative scales, given by $\eta$, remained approximately constant with $\Omega$ (Fig. 5(d)), the size of the large scales, $L_{u x}$, grew almost linearly with $U / \Omega M$ (Fig. 5(f)). The spanwise integral length scale, $L_{u y}$ also grew linearly with $U / \Omega M$ as shown in Fig. 6, however, it was much smaller than $L_{u x}$, typically within $M \pm 15 \%$.

A beneficial property of the turbulence produced by the grid was that the turbulence intensity was fairly constant while the size of the large scales $\left(L_{u x}\right)$ continued to grow by a factor of 3.5 for the range $50<U / \Omega M<175$. This suggests that for a given turbulence intensity, one can change the mean rotational rate of the wings to tailor the size of the large scales in the flow; although, we note this necessitates a change in the global isotropy (Fig. 5(c)). Previous parametric studies have not exceeded $U / \Omega M \approx 50$, and as such this is the first time this asymptotic range has been reported.

In the region where turbulence intensity and the integral scale were independent, the global isotropy, $u^{\prime} / v^{\prime}$, monotonically grew from $\sim 1.3$ to $\sim 1.7$ with increasing $R o$. In order to assess what scales are affected by this increase in anisotropy, the measured second-order structure function of the spanwise velocity fluctuations, $\left\langle(\delta v)^{2}\right\rangle \equiv\left\langle(v(x+r)-v(x))^{2}\right\rangle$, can be compared to that calculated from the structure function from the streamwise velocity fluctuations, $\left\langle(\delta u)^{2}\right\rangle$, assuming isotropy, viz,

$\left\langle(\delta v)^{2}\right\rangle_{\mathrm{cal}}=\frac{r}{2} \frac{\mathrm{d}}{\mathrm{d} r}\left\langle(\delta u)^{2}\right\rangle+\left\langle(\delta u)^{2}\right\rangle$.

This comparison is shown in Fig. 7 for one very anisotropic case V2b, where $u^{\prime} / v^{\prime}=1.41$, and three other cases (V4b, V6b, and V11b) with similar anisotropy levels near $u^{\prime} / v^{\prime}=$ 1.23. Despite the differing levels of anisotropy, all four tests have an approximately constant value of $\left\langle(\delta v)^{2}\right\rangle_{\text {cal }} /\left\langle(\delta v)^{2}\right\rangle \approx$ 1.3 for $r / \lambda \lesssim 10$. The growth of the ratio at larger scales indicates that the bulk of the anisotropy was restricted to the largest scales of the flow. While the high levels of anisotropy for some cases limit the applicability of these modes for the study of 'homogeneous, isotropic turbulence', they are nonetheless useful for tailoring turbulence for other applications where homogeneity and isotropy are not the goal.

\subsection{Influence of mean flow velocity}

Table A1 provides results for tests conducted with both solid square and solid circular wings for different $U$. For each test case, the grid was set into motion with a particular setting, and the tunnel velocity was increased holding the probes stationary at $x / M=41$. Fig. 8 shows different turbulence statistics versus the grid Reynolds number, $\operatorname{Re}_{M}$. A caveat to consider when interpreting the results shown in Fig. 8 is that the non-dimensional parameters affecting the turbulent quantities are the Reynolds number $R_{M}$ and Rossby number $R o$, as was previously found by Larssen and Devenport (2011). Clearly, both of these parameters vary when only $U$ is changed. In order to help interpret the results of Fig. 8, contour plots of the turbulent quantities as a function of both $R e$ and $R o^{-1}$ are given in Fig. 9 for the solid square wings case. The plot for the other wing geometries have the same trends and are thus not included.

The turbulence intensity appears to change with $R e_{M}$ in Fig. 8(a). This dependence of $T_{q}$ on $R e_{M}$ is atypical compared to passive grid results. Generally for passive grids, the turbulent fluctuations scale with $U$, resulting in the independence of $T_{q}$ with $\operatorname{Re}_{M}$. This is evidenced by cases E1-E7 which are plotted as triangles in Fig. 8(a). The E1-E7 cases represent the turbulence produced by the grid when it was left in the fully open, non-actuated, position. $T_{q}$ does not vary by more than $0.2 \%$ (absolute) over the same range of $R e_{M}$ that sees variations of $2.2 \%$ (absolute) for the active cases. Fig. 10(a) demonstrates that $C_{P}$ grew with $R e_{M}$ for active grid cases U8b-U14b, which are representative of all cases, while it remained approximately constant for the open grid case. The relationship between $C_{P}$ and $T_{q}$ is shown to 
be linear in Fig. 10(b) for the active case while for the open case the measurements were clustered at a single point on the $C_{P}-T_{q}$ curve. However, this perceived dependence of $T_{q}$ on $R e_{M}$ is in fact a $R o$ effect (because $U$ is in both parameters), as is evidenced by the iso-contours in Fig. 9(a) that are nearly horizontal (invariant with $R e_{M}$ ) but change drastically with $R o$.

Both Fig. 8(b) and 9(b) show that $R e_{\lambda}$ grew with $\operatorname{Re}_{M}$ as expected, however, the global isotropy in Fig. 8(c) reveals some interesting behaviour. The ratio $u^{\prime} / v^{\prime}$ became approximately constant near 1.1 for $\operatorname{Re}_{M} \geq 35,000$, except for the cases with the lowest $\Omega$ (U1-U7). This indicates that for a sufficient $R e_{M}$, the global isotropy becomes independent of $R e_{M}$ for sufficiently high wing rotation rate.

As the mean velocity was increased, the size of the dissipative scales decreased as shown in Fig. 8(d), while Fig. 9(d) confirms the weak dependence on Ro seen in Fig. 5(d) over the full range of $R_{M}$ investigated here. Conversely, as the mean velocity was increased, the size of the longitudinal energy containing scales increased (Fig. 8(f)). This length scale only has a significant dependence on $R o$ for lower values of $\Omega$ as can be seen from Fig. 5(f) and 9(f). This behaviour represents an expansion of the physical size of the scales carrying the turbulence as $R e_{M}$ increases, i.e., the large scales become larger and the small scales become smaller.

Fig. 11 plots different pre-multiplied spectra, $k F_{11}$, for constant grid settings but varying $R e_{M}$. The semi-logarithmic scale keeps the area under the curve equal to $\left\langle u^{2}\right\rangle$, which provides a true representation of the distribution of the energy over the relevant scales (e.g., Lavoie et al., 2007). For each set of constant rotational parameters in Fig. 11(a) and 11(b) the peak of the pre-multiplied spectrum is approximately invariant with $\operatorname{Re}_{M}$, both in magnitude and location. This implies that the energized wavenumbers were independent of $\operatorname{Re}_{M}$. However, this independence was no longer satisfied at the lower values of $\Omega$, as illustrated in Fig. 11(c) for cases U1b, U4b and U7b. These rotational settings are in the plateau region in Fig. 5(a), where $T_{q}$ was constant, while $u^{\prime} / v^{\prime}$ and $L_{u x}$ grew, which is consistent with the trends visible in the spectra. This demonstrates that the fundamental structure of the turbulence changes when this plateau region is reached, as the flow behaves differently than at higher $\Omega$.

\subsection{Influence of wing geometry}

Thormann and Meneveau (2014) identified that the wing geometry played a role in the produced turbulence in their study of the decay of active grid-generated turbulence with various fractal wings. However, they were unable to identify systematic trends linked to geometry. The present study allows us to comment further on the influence of wing geometry, at least at a fixed point.
For the same Rossby number, different wing shapes produced different turbulence intensities (Fig. 5(a)). The solid square wings always produced the highest intensities. The results of the solid circles and the squares with holes were very similar, but the solid circles consistently produced slightly higher levels of $T_{q}$.

The maximum blockage achievable by the solid square wings is $100 \%$, however, the probability of this happening was extremely low. The equivalent maximum possible blockage for the squares with holes and the solid circles is $79 \%$ and $78 \%$, respectively. The proximity of the frontal area of the squares with holes and the circles to each other and the small differences in their results suggests that the influence of the wing geometry was likely caused by blockage rather than the shape itself.

From the other plots in Fig. 5, except $R e_{\lambda}$, which follows the same trend as $T_{q}$, it is evident that the remaining parameters appear to be relatively independent of the wing geometry. Isotropy and the size of the scales in the flow scaled with Rossby number and not with the wing geometry. This was also true of the transverse integral length scale shown in Fig. 6. These results are confirmed by Fig. 8. Hence, the effect of the wings was to change the $T_{q}$ and $R e_{\lambda}$ and this effect is linked to their respective blockage ratio.

\section{Influence of initial conditions on spectral shape}

The active grid presents a unique opportunity for grid turbulence measurements in that the same $R e_{\lambda}$ can be produced using different sets of initial conditions over a broad range of $R e_{\lambda}$, that exceed those achievable by passive grids, and without necessitating large changes in $\operatorname{Re}_{M}$. In Fig. 12, 15 tests at 5 different $R e_{\lambda}$, spanning $177 \leq R e_{\lambda} \leq 486$, are compared. The selected test cases employ a variety of different initial conditions, including: use of two operational modes (FR and $\mathrm{CL})$, a wide range of mean rotational rates $(0.625 \mathrm{~Hz} \leq \Omega \leq$ $17.5 \mathrm{~Hz})$, all three wing geometries, and a range of grid Reynolds numbers $\left(36,000 \leq \operatorname{Re}_{M} \leq 55,000\right)$. The measurements were conducted at two different streamwise positions $(x / M=30$ and 41$)$, and with both 0.6 and $1.1 \mathrm{~mm}$ sensing length hot-wires. Hence, the test cases shown in Fig. 12 represent a broad range of the initial generating conditions and measurement conditions.

The inner variable normalized spectra of Fig. 12 illustrate that the turbulence spectra generated by different initial conditions were collapsed for a given $R e_{\lambda}$ in the range $k \eta>10^{-3}$. The variation of the scaling exponent, $k^{m}$, in the scaling range is always less than $\pm 1.2 \%$ for a given $R e_{\lambda}$. The scaling exponent did display a dependence on $R e_{\lambda}$ as it changed from $m=-1.42$ to -1.56 over the investigated range. The lower limit of which is near, but not exactly, Kolmogrov's $k^{-5 / 3}$ prediction. This is in general agreement with the trends found by Mydlarski and Warhaft (1996). The 
scaling exponent, $m$, was measured over $0.004 \leq k \eta \leq 0.05$, which was found to be within the scaling range for all active grid test cases. From the illustrated spectra in Fig. 12 and the minimal variations in $m$, it is clear that the spectra were well collapsed in the scaling and dissipative ranges at a given $R e_{\lambda}$ for the active grid.

The spectra at constant $R e_{\lambda}$ only differ from one-another at low wavenumbers, which are associated with the large scales. For instance, at $R e_{\lambda} \approx 485$, case U7b $(\Omega \pm \omega=0.625 \pm$ $0.375 \mathrm{~Hz}$ ) has a broadband peak at low wavenumbers that differs from U14b $(\Omega \pm \omega=3 \pm 2 \mathrm{~Hz})$. This difference is associated with the dependence of the energy peak on $\Omega$, as indicated in Fig. 11. By comparison of the results presented in Table A1, these two test cases have approximately the same $\eta$ and $\lambda$, but $L_{u x}$ for U7b was nearly twice that of U14b. The integral scale is a large scale parameter. Furthermore, the global isotropy, which is also a large scale parameter, for U7b was $u^{\prime} / v^{\prime}=1.46$, while it was 1.12 for U14b. These comparisons quantitively illustrate that the spectra and flow differ only at the large scales, while their small scales remain approximately the same for a given $R e_{\lambda}$.

These results suggest that for these $R e_{\lambda}$ and reasonably homogeneous and isotropic turbulence, the structure of the turbulence itself has predominately 'forgotten' its initial conditions, and any remnants of them are carried only in the largest scales.

\section{Conclusions}

A new active grid with a greater number of elements across the tunnel cross-section and that has decoupled the motion of adjacent wings was used to extend earlier parametric studies to include higher Rossby numbers, $R o=U / \Omega M$, and different wing geometries. The three parameters shown to have the greatest influence on the produced turbulence were $R o, R e_{M}$, and wing blockage. It was shown that for $R o>$ 50 , an asymptotic state was reached in turbulence intensity and $R e_{\lambda}$ whereby increasing $R o$ further had no impact on these properties. However, if Ro was increased by decreasing $\Omega$ and holding $U$ constant, then anisotropy and $L_{u x}$ both grew. Hence, in this asymptotic state, a certain degree of control authority could be exerted over the size of the largest scales for a given turbulence intensity, provided a loss in isotropy was acceptable. For moderate and high $\Omega$, the global isotropy reached an asymptote near $u^{\prime} / v^{\prime}=1.1$ for $R e_{M}>35,000$. The effect of the anisotropy, felt mostly for low values of $\Omega$, was shown to be contained primarily in scales larger than $10 \lambda$. Consistent with this observation, both the Kolmogorov and Taylor microscales were only weakly dependent on $R o$ and primarily changed with $R e_{M}$. Solid square wings consistently produced the highest turbulence intensities and $R e_{\lambda}$, while circular wings and square wings with holes produced lower values. This was linked to the effective maximum blockage of the various wing geometries. It was further identified that the isotropy, and size of the scales was relatively independent of wing geometry.

It was also observed that the high wavenumber spectra were nearly identical for approximately homogeneous turbulence at a given $R e_{\lambda}$. This was verified for turbulence at the same $R e_{\lambda}$, over the range $177 \leq R e_{\lambda} \leq 486$, produced with considerably different active grid conditions. This suggests that the small scale turbulence organization is approximately independent of its initial conditions for a given $R e_{\lambda}$ in homogeneous and approximately isotropic turbulence, in agreement with theoretical expectations. Furthermore, it was identified that any differences that do remain in the produced turbulence spectrum are isolated to the largest scales, and carried in ensemble parameters such $u^{\prime} / v^{\prime}$ and $L_{u x}$.

Acknowledgements This project was funded through a Natural Sciences and Engineering Research Council of Canada (NSERC) Engage grant in collaboration with Anemoi Technologies Inc., specialists in simulation and test facilities. RJH acknowledges financial support from the Ontario Provincial Government and NSERC. The authors would like to thank Mr. S. Ciurzynski of Anemoi Technologies Inc. who aided in the design and manufacturing of the active grid. Special appreciation is given to Mr. R. Santos Baptista who aided with the data acquisition, and to Ms. E. Dogan and Prof. B. Ganapathisubramani for insightful comments on the spectra.

\section{References}

L. H. Benedict and R. D. Gould. Towards better uncertainty estimates for turbulence statistics. Exp. Fluids, 22:129136, 1996.

G. P. Bewley, J. Kassel, and E. Bodenschatz. Control of turbulence with a high degree-of-freedom active grid. In 14th European Turbulence Conference, 2013.

E. Bodenschatz, G. P. Bewley, H. Nobach, M. Sinhuber, and $\mathrm{H}$. Xu. Variable density turbulence tunnel facility. Rev. Sci. Inst., 85(093908), 2014.

P. Burattini and R. A. Antonia. The effect of different X-wire calibration schemes on some turbulence statistics. Exp. Fluids, 38:80-89, 2005.

R. B. Cal, J. Lebrón, L. Castillo, H. S. Kang, and C. Meneveau. Experimental study of the horizontally averaged flow structure in a model wind-turbine array boundary layer. J. Renewable Sustain. Energy, 2(013106), 2010.

H. E. Cekli and W. van de Water. Tailoring turbulence with an active grid. Exp. Fluids, 49:409-416, 2010.

G. Comte-Bellot and S. Corrsin. The use of a contraction to improve the isotropy of grid-generated turbulence. J. Fluid Mech., 25(4):657-682, 1966.

G. Comte-Bellot and S. Corrsin. Simple Eulerian time correlation of full- and narrow-band velocity signals in gridgenerated, 'isotropic' turbulence. J. Fluid Mech., 48:273337, 1971 . 
R. J. Hearst and P. Lavoie. Decay of turbulence generated by a square-fractal-element grid. J. Fluid Mech., 741:567584, 2014.

R. J. Hearst, O. R. H. Buxton, B. Ganapathisubramani, and P. Lavoie. Experimental estimation of fluctuating velocity and scalar gradients in turbulence. Exp. Fluids, 53(4): 925-942, 2012.

J. C. Isaza, R. Salazar, and Z. Warhaft. On grid-generated turbulence in the near- and far field regions. J. Fluid Mech., 753:402-426, 2014.

F. E. Jørgensen. How to measure turbulence with hotwire anemometers - a practical guide. Dantec Dynamics, 2002.

H.S. Kang, S. Chester, and C. Meneveau. Decaying turbulence in an active-grid-generated flow and comparisons with large-eddy simulation. J. Fluid Mech., 480:129-160, 2003.

P. Knebel, A. Kittel, and J. Peinke. Atmospheric wind field conditions generated by active grids. Exp. Fluids, 51: 471-481, 2011.

A. N. Kolmogorov. The local structure of turbulence in incompressible viscous fluids for very large Reynolds numbers. Dokl. Akad. Nauk SSSR, 30(4):301-305, 1941.

J. V. Larssen and W. J. Devenport. On the generation of large-scale homogeneous turbulence. Exp. Fluids, 50: 1207-1223, 2011.

P. Lavoie, L. Djenidi, and R. A. Antonia. Effects of initial conditions in decaying turbulence generated by passive grids. J. Fluid Mech., 585:395-420, 2007.

H. Makita. Realization of a large-scale turbulence field in a small wind tunnel. Fluid Dyn. Res., 8:53-64, 1991.

J. Mi, R. C. Deo, and G. J. Nathan. Fast-convergence iterative scheme for filtering velocity signals and finding Kolmogorov scales. Phys. Rev. E, 71(066304), 2005.

J. Mi, M. Xu, and C. Du. Digital filter for hot-wire measurements of small-scale turbulence properties. Meas. Sci. Tech., 22(125401), 2011.

I. S. Miller, D. A. Shah, and R. A. Antonia. A constant temperature hot-wire anemometer. J. Phys. E: Sci. Instrum., 20:311-314, 1987.

L. Mydlarski and Z. Warhaft. On the onset of highReynolds-number grid-generated wind tunnel turbulence. J. Fluid Mech., 320:331-368, 1996.

L. Mydlarski and Z. Warhaft. Passive scalar statistics in high-Péclet-number grid turbulence. J. Fluid Mech., 358: $135075,1998$.

R.E.G. Poorte and A. Biesheuvel. Experiments on the motion of gas bubbles in turbulence generated by an active grid. J. Fluid Mech., 461:127-154, 2002.

N. Sharp, S. Neuscamman, and Z. Warhaft. Effects of large-scale free stream turbulence on a turbulent boundary layer. Phys. Fluids, 21(095105), 2009.
M. J. Sytsma and L. Ukeiley. Mean loads from wind-tunnel turbulence on low-aspect-ratio flat plates. J. Aircraft, 50 (3):863-870, 2013.

A. Thormann and C. Meneveau. Decay of homogeneous, nearly isotropic turbulence behind active fractal grids. Phys. Fluids, 26(025112), 2014.

J. C. Wyngaard. Measurements of small-scale turbulence structure with hot wires. J. Sci. Instr., 1(2):1105-1108, 1968.

Y. Zhu and R. A. Antonia. The spatial resolution of hotwire arrays for the measurement of small-scale turbulence. Meas. Sci. Tech., 7:1349-1359, 1996. 


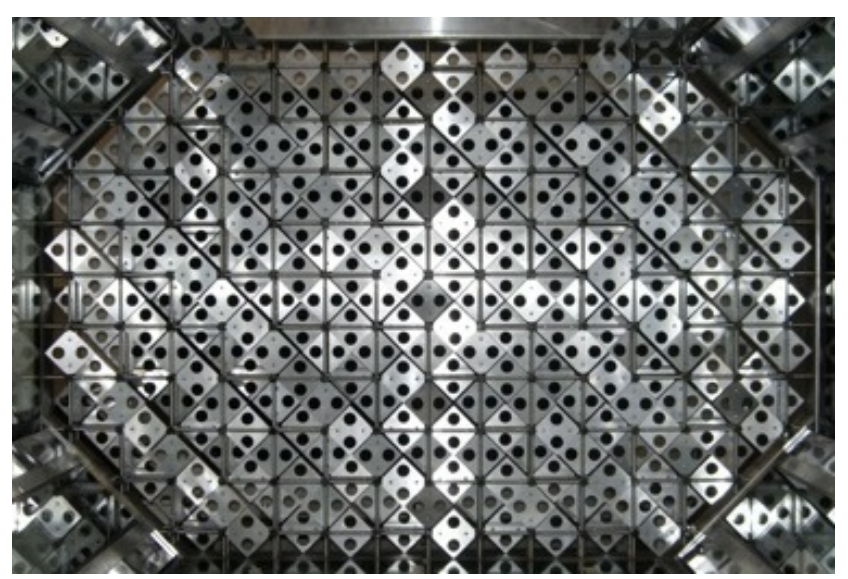

(a)

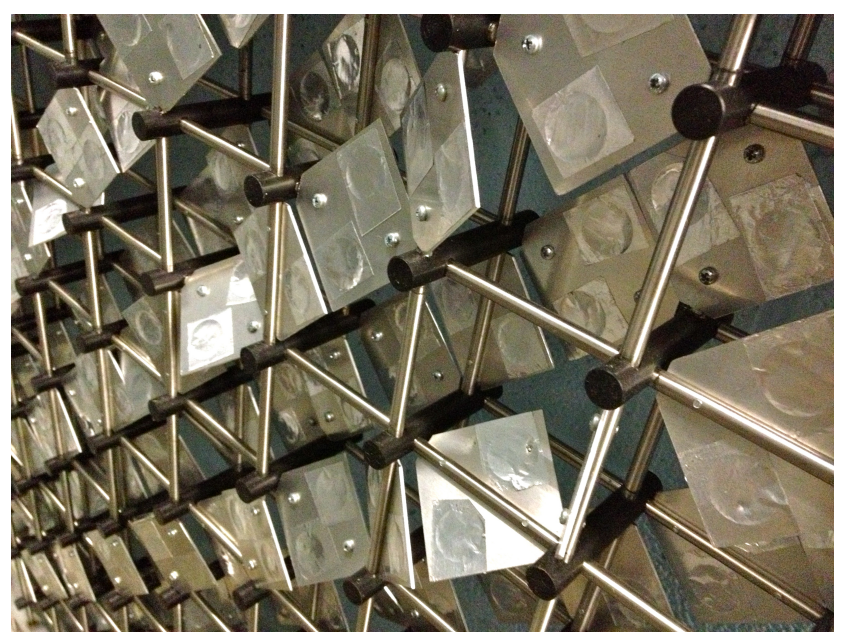

(b)

Fig. 1: Photographs of the active grid (a) in situ and (b) showing the double-mesh design.

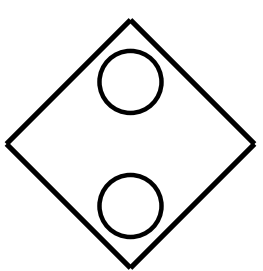

(a)

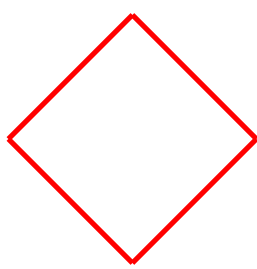

(b)

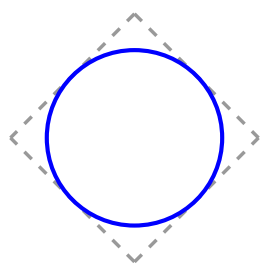

(c)

Fig. 2: Schematics of the active grid wing geometries: (a) $55 \mathrm{~mm} \times 55 \mathrm{~mm}$ squares with dia. $20 \mathrm{~mm}$ holes, (b) $55 \mathrm{~mm} \times 55 \mathrm{~mm}$ solid squares, and (c) dia. $55 \mathrm{~mm}$ circles. 

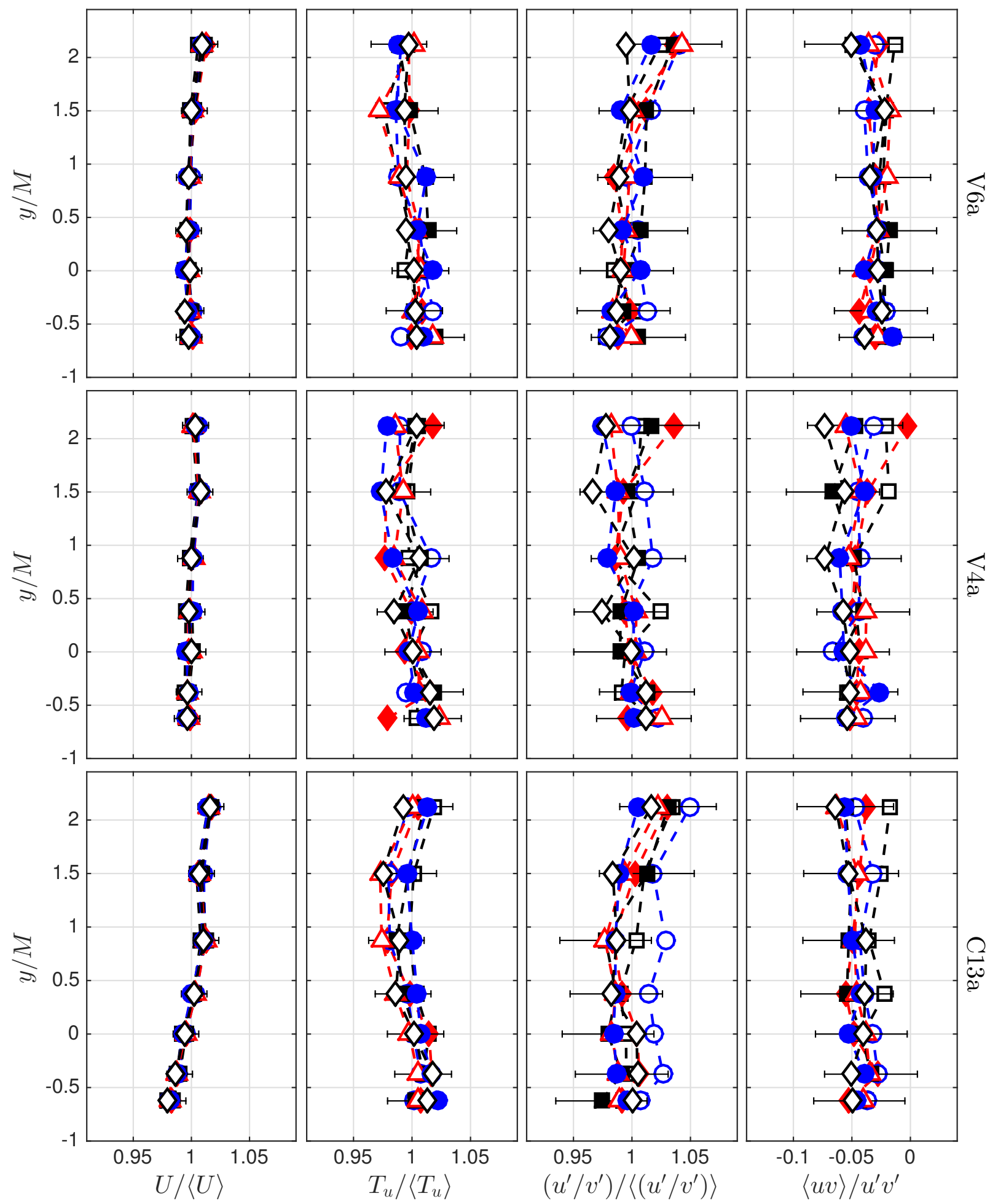

Fig. 3: Homogeneity profiles of the (from left to right) mean velocity, streamwise turbulence intensity, global isotropy, and normalised Reynolds shear stress, for test cases (from top to bottom) V6a, V4a, and C13a. All scans were performed at $x / M=30$. (口) $z / M=-11 / 8,(\diamond) z / M=-7 / 8,(\bigcirc) z / M=-3 / 8,(\square) z / M=0,(\triangle) z / M=3 / 8,(\bullet) z / M=7 / 8,(\diamond) z / M=11 / 8$. 


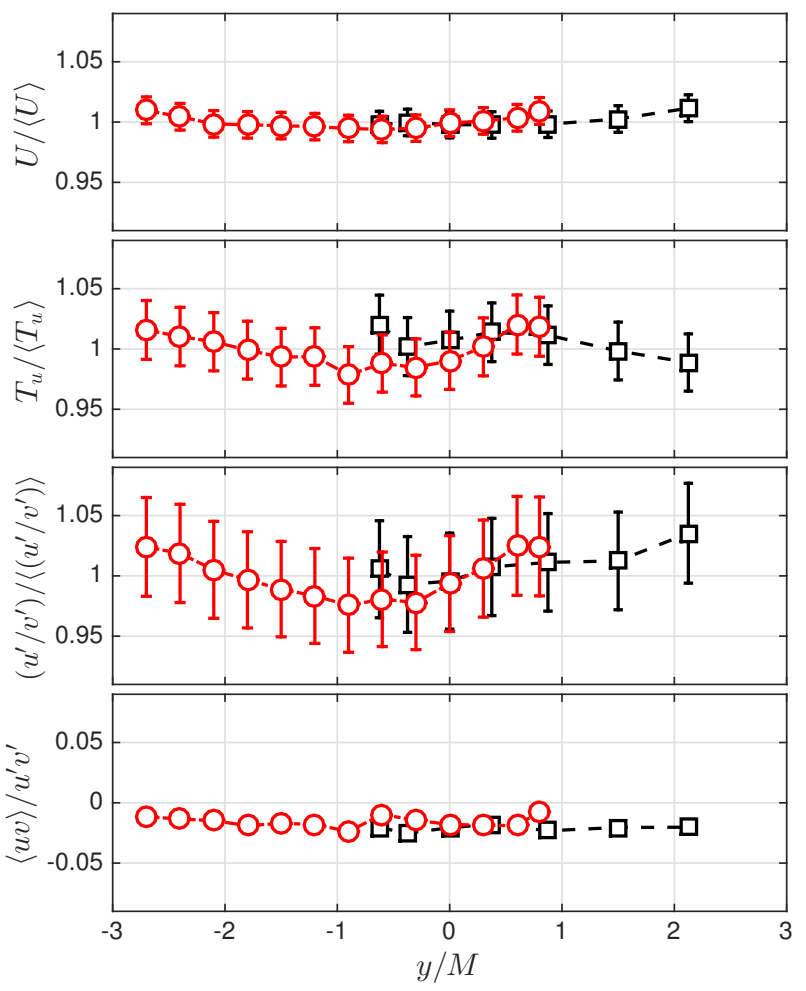

Fig. 4: Homogeneity scan over an extended range for test case V6a. ( $\bigcirc$ ) from transverse integral scale measurements, (口) from $z / M=0$ axis of planar homogeneity scans. 


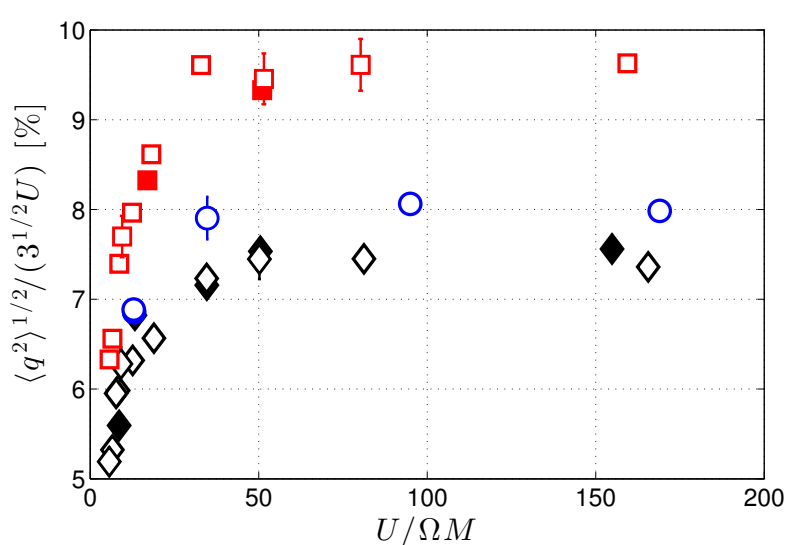

(a)

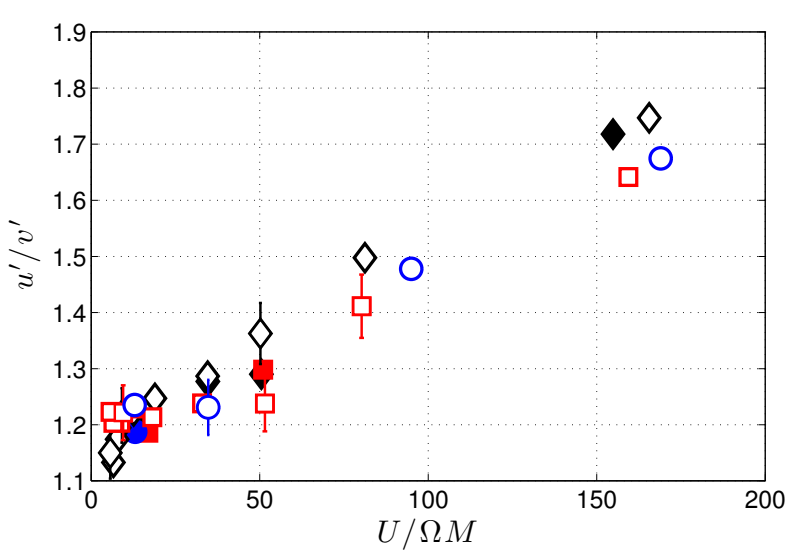

(c)

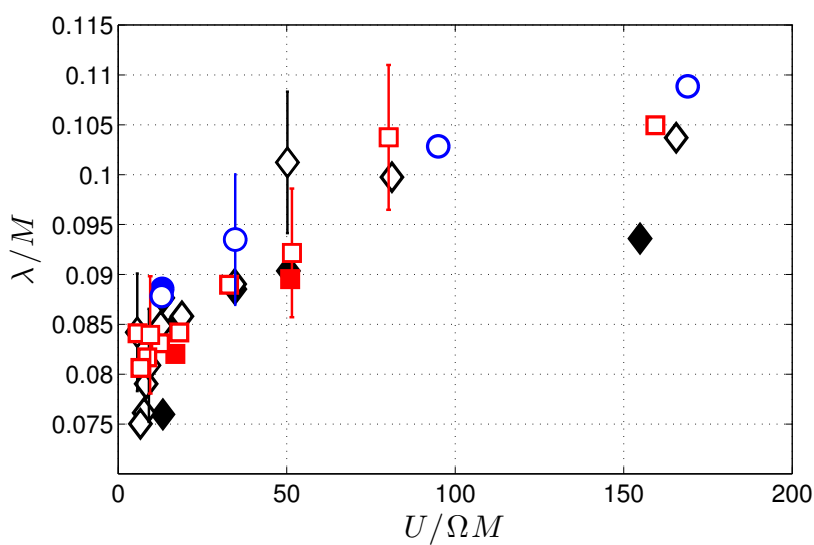

(e)

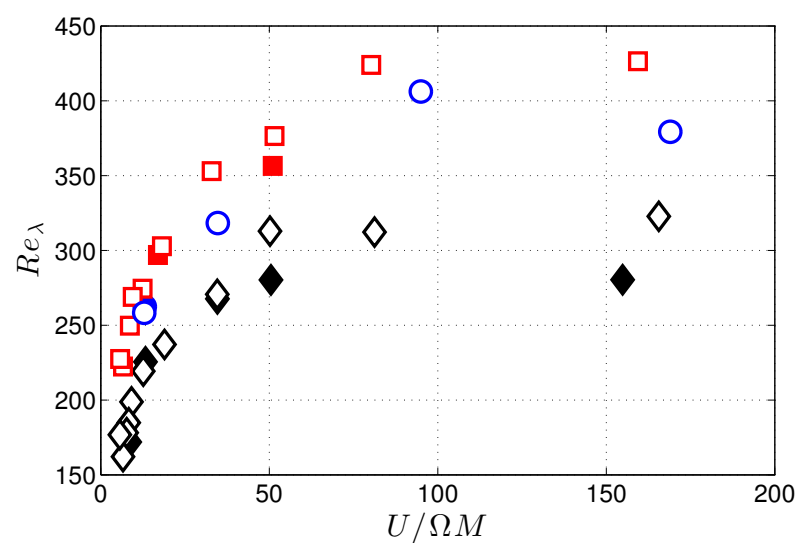

(b)

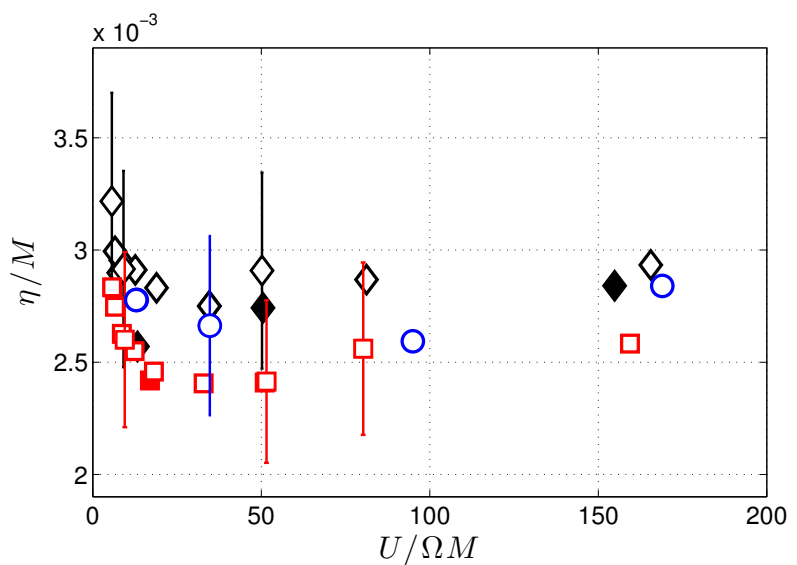

(d)

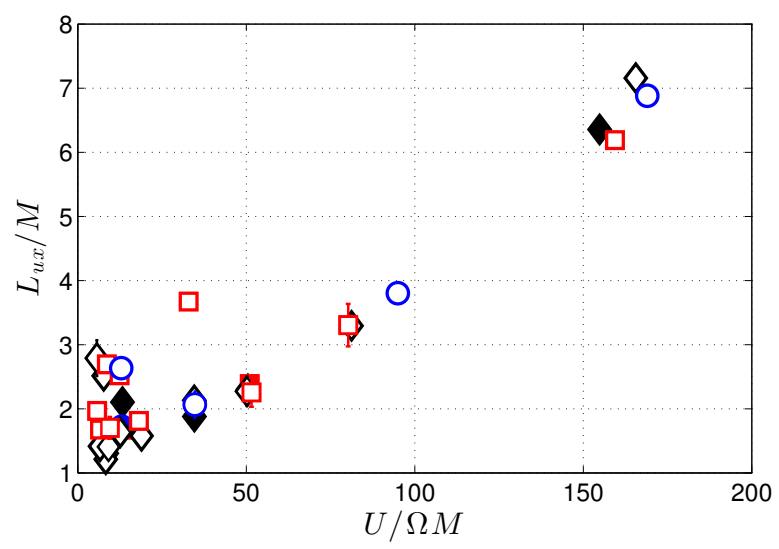

(f)

Fig. 5: Variation of turbulence properties with Rossby number, $R o=U / \Omega M$. In all cases here, $\Omega$ was the only quantity varied in $R o$, i.e., both $U$ and $M$ were held constant. $(\diamond)$ square wings with holes, $(\square)$ solid square wings, $(\bigcirc)$ solid circular wings; empty symbols are tests in FR mode, and filled symbols are tests in CL mode. Test cases included here are V1-V11a,b,c and T1a,b. Error bars illustrate the typical uncertainty associated with the measurement of these parameters. 


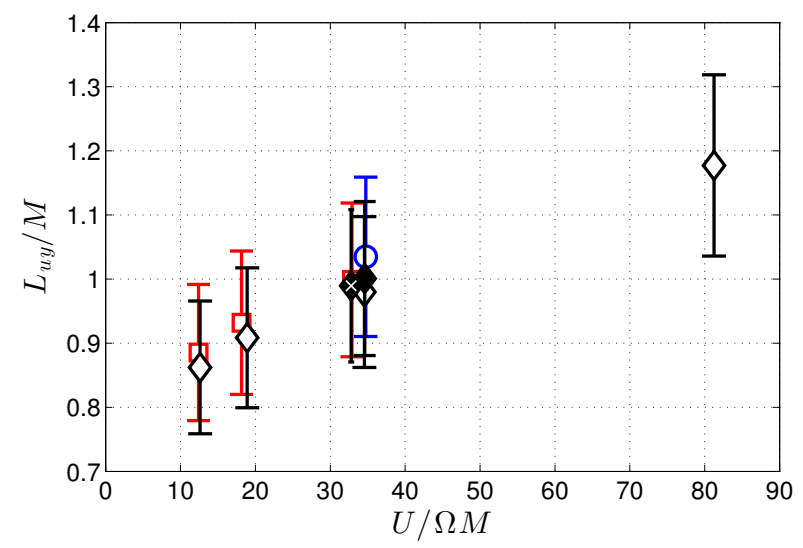

Fig. 6: Spanwise integral length scale, $L_{u y}$, for changing Rossby number, $R o=U / \Omega M$. In all cases here, $\Omega$ was the only quantity varied in $R o$, i.e., both $U$ and $M$ were held constant. $(\diamond)$ square wings with holes, $(\square)$ solid square wings, (○) solid circular wings; empty symbols are tests in FR mode, filled symbols are tests in CL mode, and filled symbols with a ' $X$ ' are tests in 10CL mode.

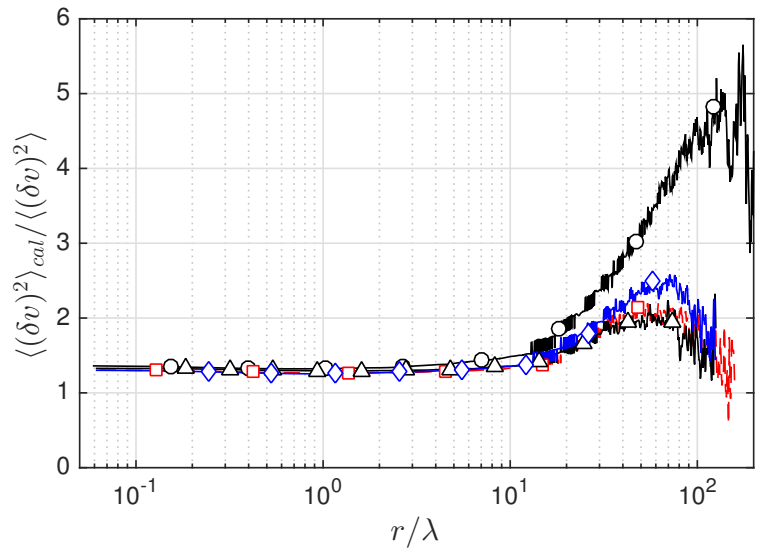

Fig. 7: Ratio of the estimated isotropic transverse secondorder structure function to the measured transverse secondorder structure function. $(\bigcirc) \mathrm{V} 2 \mathrm{~b}, u^{\prime} / v^{\prime}=1.41$; $(\diamond) \mathrm{V} 4 \mathrm{~b}$, $u^{\prime} / v^{\prime}=1.24$; (口) V6b, $u^{\prime} / v^{\prime}=1.23 ;(\triangle) \mathrm{V} 11 \mathrm{~b}, u^{\prime} / v^{\prime}=1.22$. 


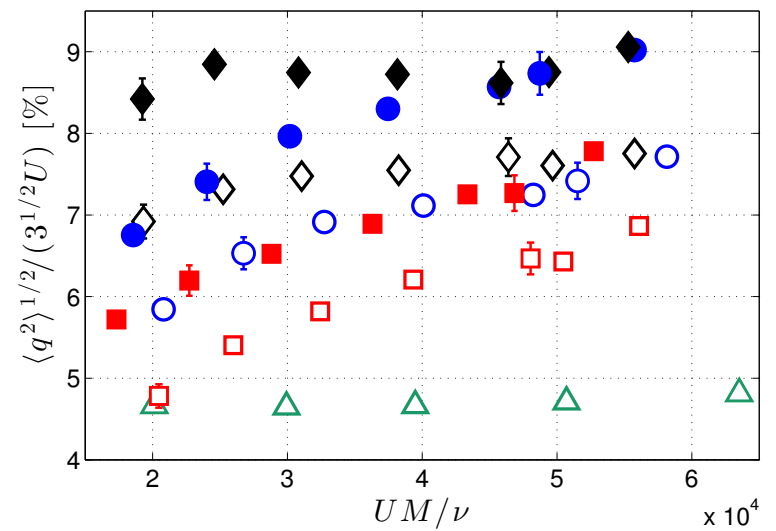

(a)

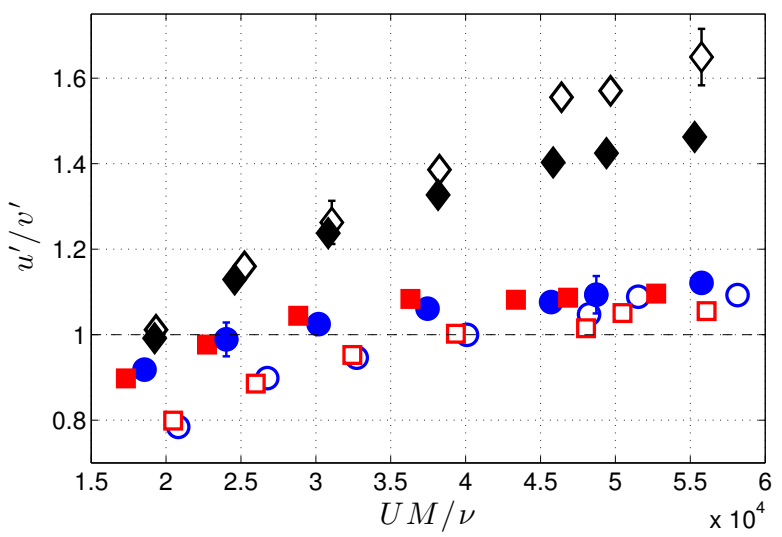

(c)

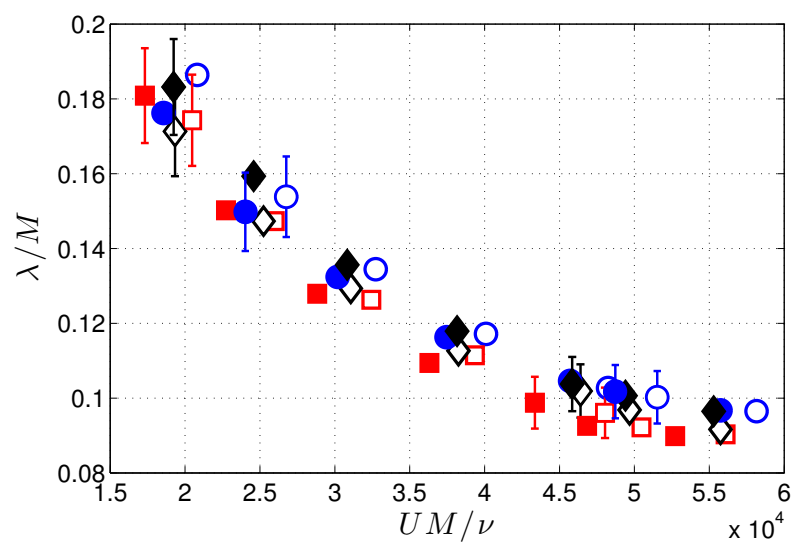

(e)

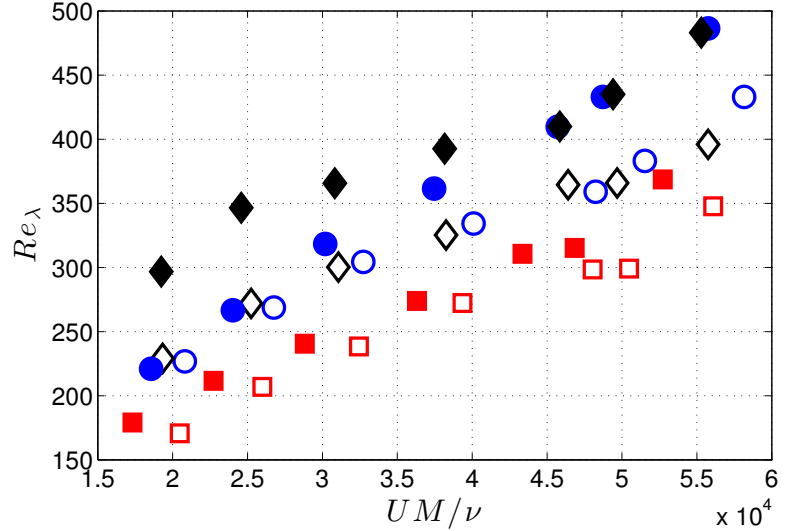

(b)

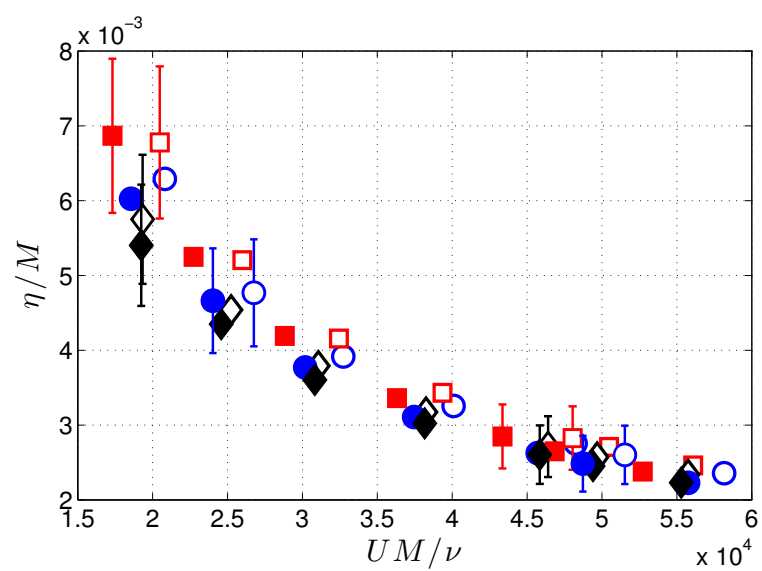

(d)

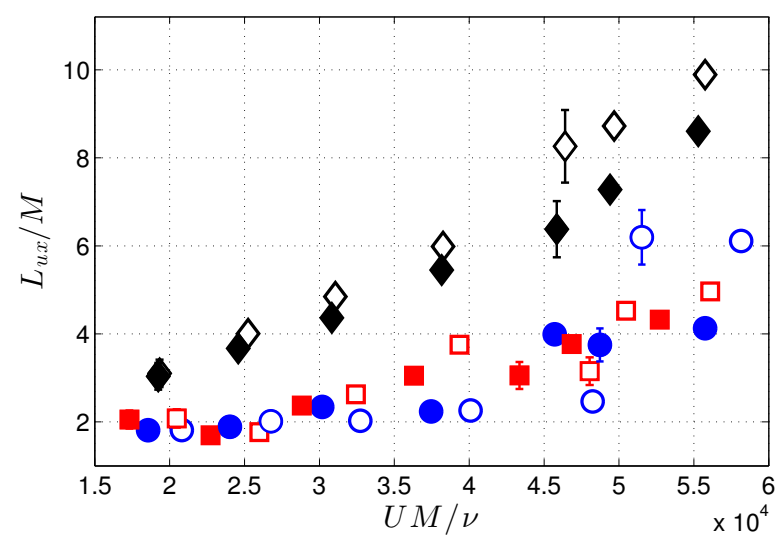

(f)

Fig. 8: Relationship between turbulence properties and the grid Reynolds number, $\operatorname{Re}_{M}=U M / v$. For each case shown, $U$ was the only quantity varied. $(\diamond) \mathrm{U} 1-\mathrm{U} 7,(\bigcirc) \mathrm{U} 8-\mathrm{U} 14,(\square) \mathrm{U} 15-\mathrm{U} 21,(\triangle)$ open grid cases E1-E7 offset by $+3 \%$; filled symbols represents solid square wings ('b' cases), and empty symbols represent solid circular wings ('c' cases). 


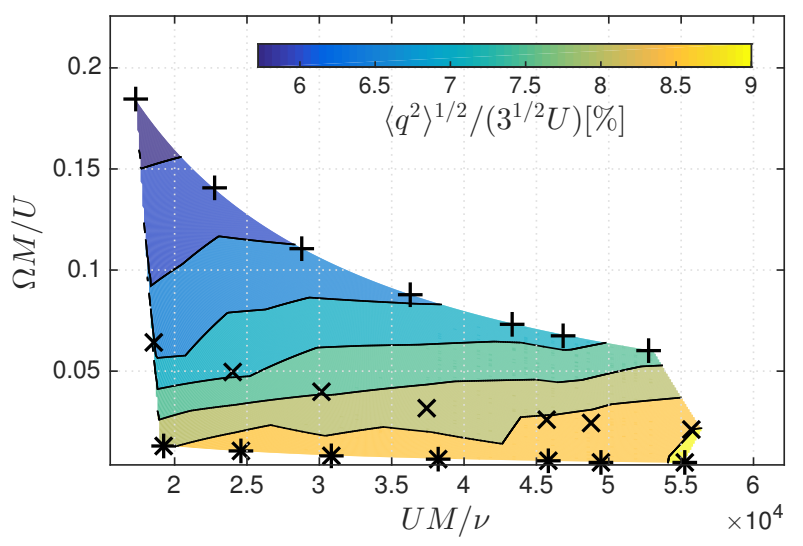

(a)

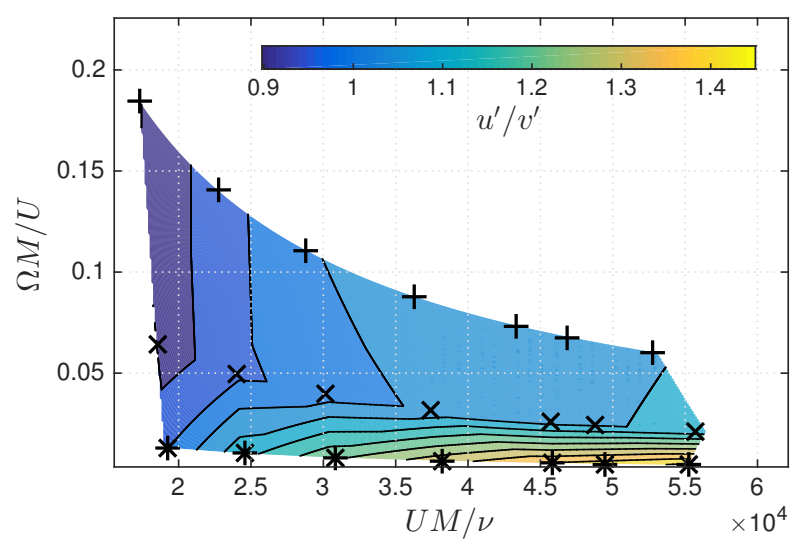

(c)

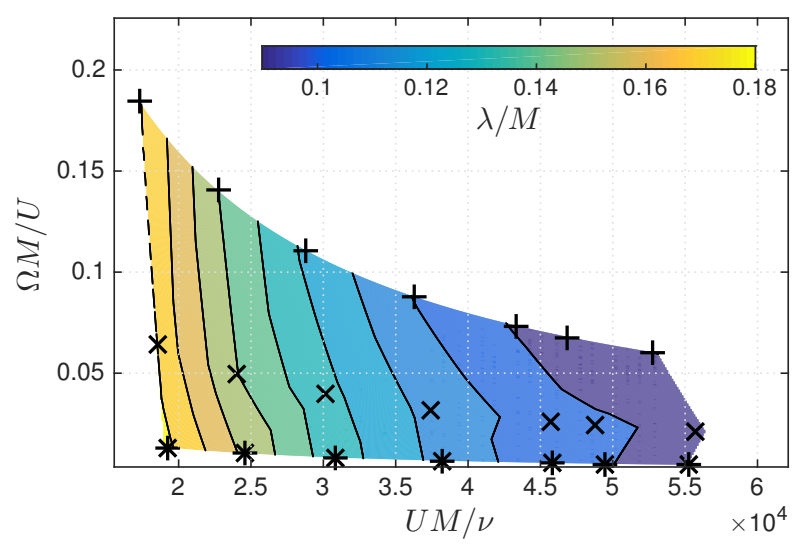

(e)

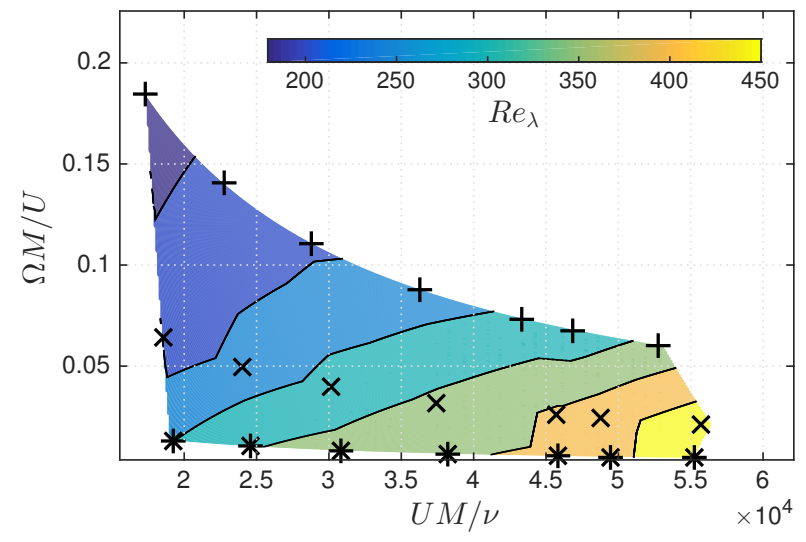

(b)

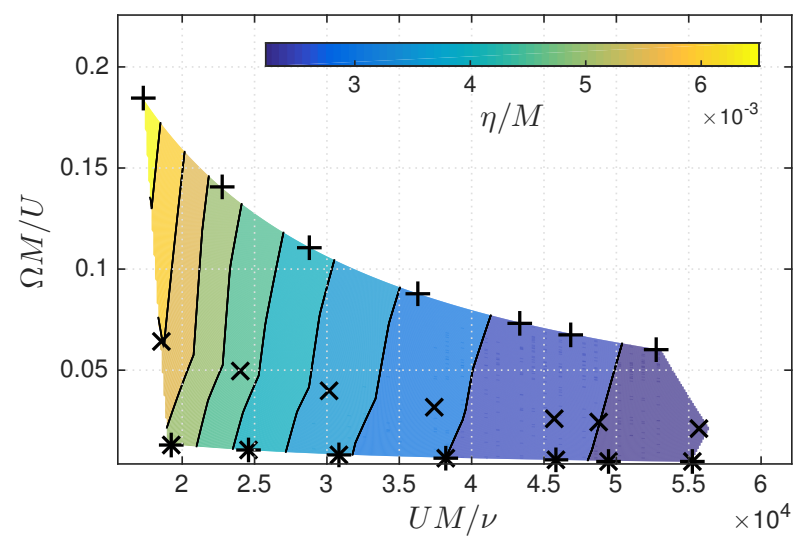

(d)

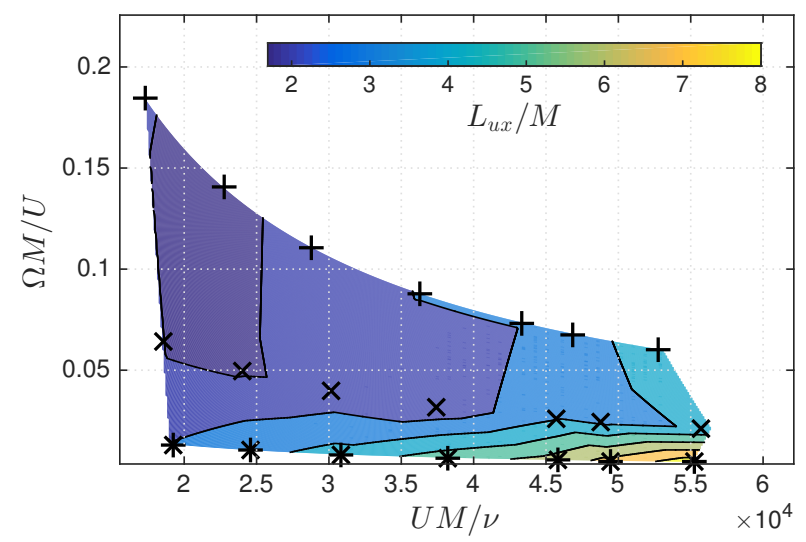

(f)

Fig. 9: Contours identifying the co-dependence of the produced turbulence on the Reynolds number and the Rossby number (plotted in inverse). Contours are drawn for solid square wings. (*) U1b to U7b, $\Omega \pm \omega=0.625 \pm 0.375 \mathrm{~Hz} ;(\times) \mathrm{U} 8 \mathrm{~b}$ to U14b, $\Omega \pm \omega=3 \pm 2 \mathrm{~Hz}$; (+) U15b to U21b, $\Omega \pm \omega=8 \pm 7 \mathrm{~Hz}$. 


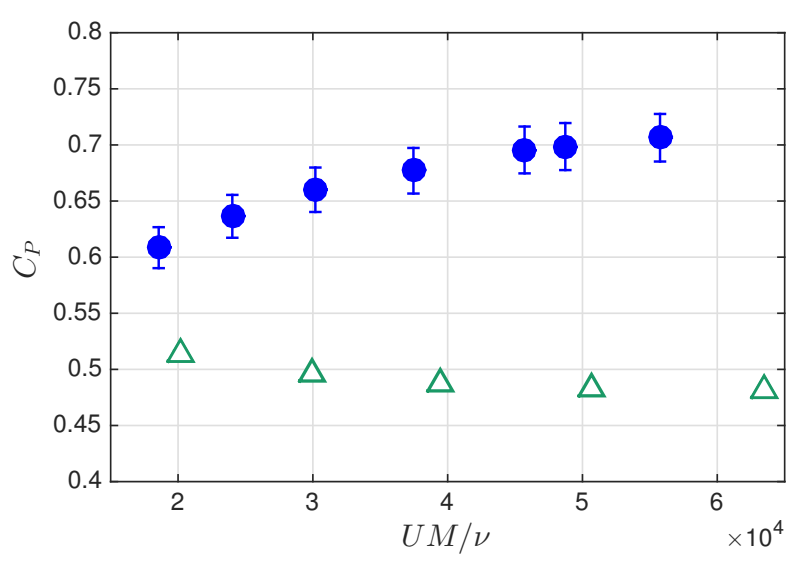

(a)

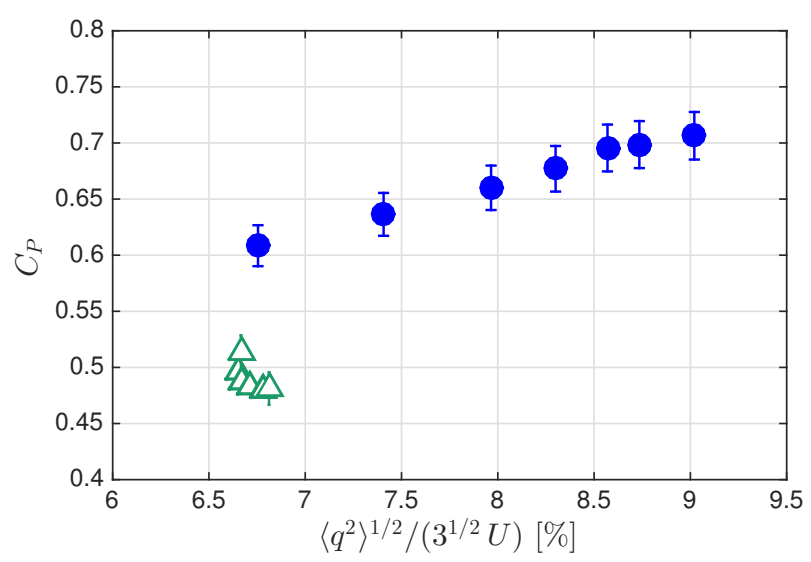

(b)

Fig. 10: Non-dimensional pressure drop across the grid compared to (a) grid Reynolds number, and (b) turbulence intensity. For each case shown, $U$ was the only quantity varied. $(\bullet)$ U8b-U14b, $(\triangle)$ open grid cases E1-E7 with $C_{p}$ offset by +0.4 and $T_{q}$ offset by $+5 \%$.

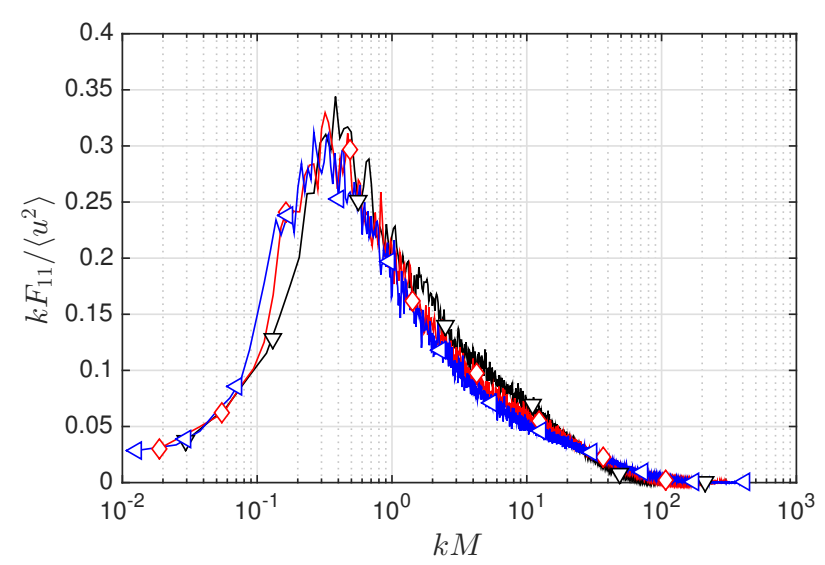

(a)

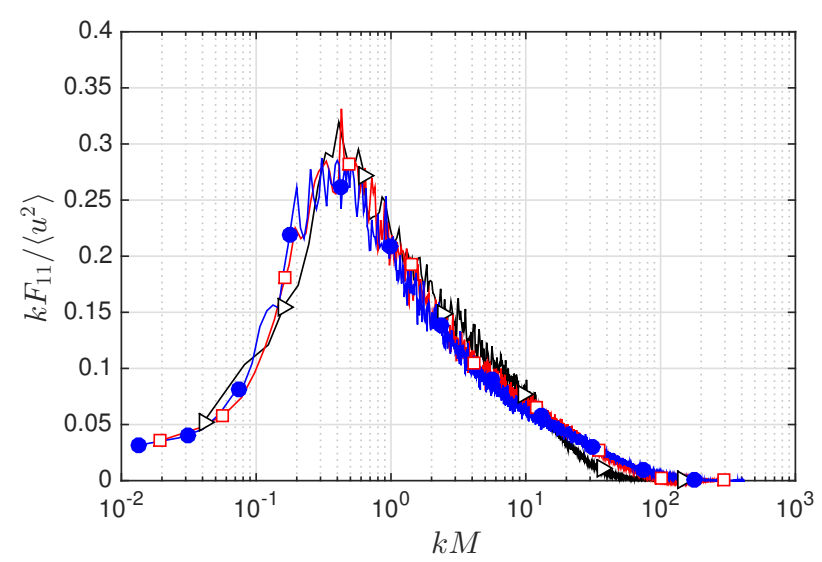

(b)

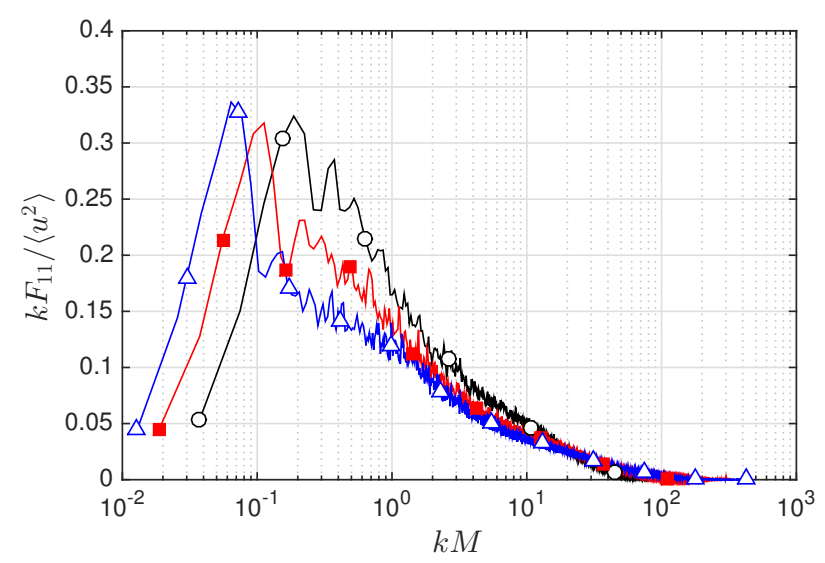

(c)

Fig. 11: Pre-multiplied spectra comparing cases with the same grid parameters but different $R e_{M}$. (a) Cases with $\Omega \pm \omega=3 \pm 2 \mathrm{~Hz}:(\nabla) \mathrm{U} 9 \mathrm{~b}, \operatorname{Re}_{M}=24,000$; $(\diamond) \mathrm{U} 11 \mathrm{~b}$ $R e_{M}=37,500$; $\triangleleft$ ) U14b, $\operatorname{Re}_{M}=55,800$. (b) Cases with

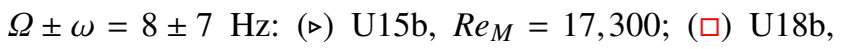
$R e_{M}=36,300$; (•) U21b, $R e_{M}=52,700$. (c) Cases with $\Omega \pm \omega=0.625 \pm 0.375 \mathrm{~Hz}:(\bigcirc) \mathrm{U} 1 \mathrm{~b}, \operatorname{Re}_{M}=19,200$; (口) U4b, $R e_{M}=38,200 ;(\triangle) \mathrm{U} 7 \mathrm{~b}, \operatorname{Re}_{M}=55,300$ 


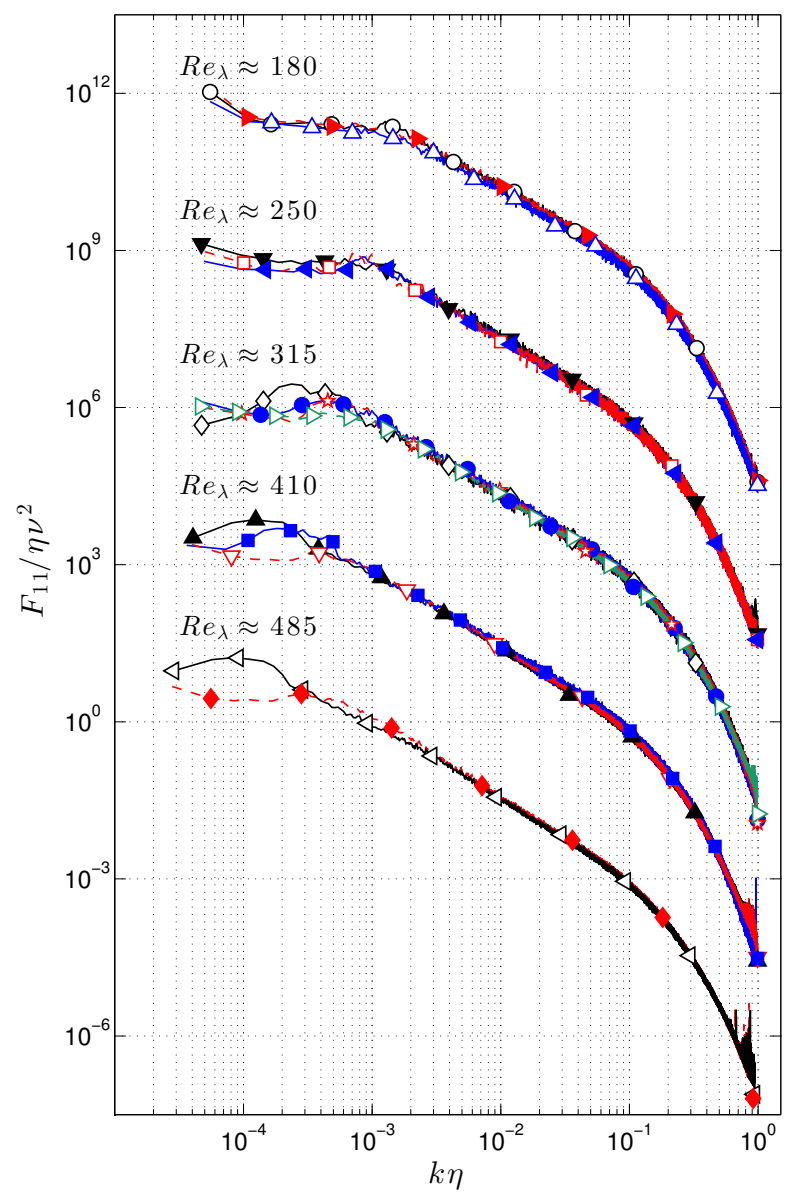

Fig. 12: Inner variable normalized spectra at the same $R e_{\lambda}$ but produced by different initial conditions. Each set of curves is offset by $10^{-3}$, starting from the $R e_{\lambda} \approx 180$ case. (○) V8a, $R e_{\lambda}=185$; ( ) V9a, $R e_{\lambda}=178 ;(\triangle) \mathrm{V} 11 \mathrm{a}, R e_{\lambda}=$ 177; ( $\nabla) \mathrm{V} 8 \mathrm{~b}, R e_{\lambda}=250$; (口) C13a, $R e_{\lambda}=250$; (ঊ) A7a, $R e_{\lambda}=249 ;(\diamond) \mathrm{V} 2 \mathrm{a}, R e_{\lambda}=312 ;$ ( $\left(\right.$ ) V3a, $R e_{\lambda}=312$;

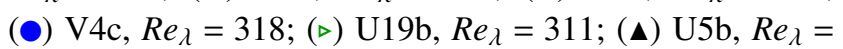

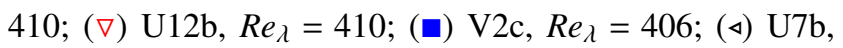
$R e_{\lambda}=483 ;(\diamond) \mathrm{U} 14 \mathrm{~b}, R e_{\lambda}=486$. 
Appendix A: Supplemental parametric study tables

Table A1: The effect of mean velocity on the produced turbulence. Measurements performed at $x / M=41$.

\begin{tabular}{|c|c|c|c|c|c|c|c|c|c|c|c|c|}
\hline Case & Mode & Ro & $\begin{array}{r}R e_{M} \\
\times 10^{3} \\
\end{array}$ & $\begin{array}{c}\Omega \pm \omega \\
{[\mathrm{Hz}]}\end{array}$ & $\begin{array}{c}U \\
{[\mathrm{~m} / \mathrm{s}]}\end{array}$ & $\begin{array}{c}T_{q} \\
{[\%]} \\
\end{array}$ & $R e_{\lambda}$ & $u^{\prime} / v^{\prime}$ & $\eta / M$ & $\lambda / M$ & $L_{u x} / M$ & $C_{P}$ \\
\hline U1b & $\overline{\mathrm{FR}}$ & 76 & 18.4 & $0.625 \pm 0.375$ & 3.8 & 8.4 & 297 & 0.99 & 0.0054 & 0.183 & 3.04 & $\overline{0.64}$ \\
\hline $\mathrm{U} 2 \mathrm{~b}$ & FR & 98 & 23.7 & $0.625 \pm 0.375$ & 4.9 & 8.8 & 346 & 1.13 & 0.0043 & 0.159 & 3.67 & 0.67 \\
\hline $\mathrm{U} 3 \mathrm{~b}$ & FR & 122 & 29.5 & $0.625 \pm 0.375$ & 6.1 & 8.7 & 366 & 1.24 & 0.0036 & 0.136 & 4.36 & 0.70 \\
\hline $\mathrm{J} 4 \mathrm{~b}$ & FR & 152 & 36.8 & $0.625 \pm 0.375$ & 7.6 & 8.7 & 393 & 1.33 & 0.0030 & 0.118 & 5.45 & 0.72 \\
\hline $\mathrm{J} 5 \mathrm{~b}$ & FR & 182 & 44.1 & $0.625 \pm 0.375$ & 9.1 & 8.6 & 410 & 1.40 & 0.0026 & 0.104 & 6.38 & 0.74 \\
\hline $\mathrm{J} 6 \mathrm{~b}$ & FR & 198 & 47.9 & $0.625 \pm 0.375$ & 9.9 & 8.8 & 435 & 1.42 & 0.0025 & 0.101 & 7.28 & 0.74 \\
\hline $\mathrm{U} 7 \mathrm{~b}$ & FR & 222 & 53.8 & $0.625 \pm 0.375$ & 11.1 & 9.1 & 483 & 1.46 & 0.0022 & 0.096 & 8.60 & 0.76 \\
\hline$\overline{\mathrm{U}} 8 \mathrm{~b}$ & FR & 15 & 17.9 & $3.0 \pm 2.0$ & 3.7 & 6.8 & 221 & 0.92 & 0.0060 & 0.176 & 1.81 & 0.61 \\
\hline $\mathrm{J} 9 \mathrm{~b}$ & FR & 20 & 23.2 & $3.0 \pm 2.0$ & 4.8 & 7.4 & 267 & 0.99 & 0.0047 & 0.150 & 1.89 & 0.64 \\
\hline $\mathrm{J} 10 \mathrm{~b}$ & FR & 25 & 29.5 & $3.0 \pm 2.0$ & 6.1 & 8.0 & 318 & 1.02 & 0.0038 & 0.132 & 2.34 & 0.66 \\
\hline $11 \mathrm{~b}$ & FR & 32 & 36.8 & $3.0 \pm 2.0$ & 7.6 & 8.3 & 362 & 1.06 & 0.0031 & 0.116 & 2.24 & 0.68 \\
\hline$J 12 b$ & FR & 38 & 44.6 & $3.0 \pm 2.0$ & 9.2 & 8.6 & 410 & 1.08 & 0.0026 & 0.105 & 3.99 & 0.70 \\
\hline $\mathrm{J} 13 \mathrm{~b}$ & FR & 41 & 47.9 & $3.0 \pm 2.0$ & 9.9 & 8.7 & 433 & 1.09 & 0.0025 & 0.102 & 3.75 & 0.70 \\
\hline $\mathrm{U} 14 \mathrm{~b}$ & FR & 47 & 54.7 & $3.0 \pm 2.0$ & 11.3 & 9.0 & 486 & 1.12 & 0.0022 & 0.097 & 4.13 & 0.71 \\
\hline $15 b$ & $\overline{F R}$ & 5 & 17.0 & $8.0 \pm 7.0$ & 3.5 & 5.7 & 179 & 0.90 & 0.0069 & 0.181 & 2.05 & $\overline{0.70}$ \\
\hline $\mathrm{J} 16 \mathrm{~b}$ & FR & 7 & 22.3 & $8.0 \pm 7.0$ & 4.6 & 6.2 & 212 & 0.98 & 0.0052 & 0.150 & 1.69 & 0.70 \\
\hline $17 b$ & FR & 9 & 28.1 & $8.0 \pm 7.0$ & 5.8 & 6.5 & 241 & 1.04 & 0.0042 & 0.128 & 2.37 & 0.72 \\
\hline $18 b$ & FR & 11 & 35.4 & $0 \pm 7.0$ & 7.3 & 6.9 & 274 & 1.08 & 0.0034 & 0.109 & 3.05 & 0.73 \\
\hline $\mathrm{J} 19 \mathrm{~b}$ & FR & 14 & 42.1 & $8.0 \pm 7.0$ & 8.7 & 7.3 & 311 & 1.08 & 0.0028 & 0.099 & 3.05 & 0.74 \\
\hline $20 b$ & FR & 15 & 45.5 & $8.0 \pm 7.0$ & 9.4 & 7.3 & 315 & 1.09 & 0.0026 & 093 & 3.77 & 0.74 \\
\hline $\mathrm{U} 21 \mathrm{~b}$ & FR & 17 & 51.8 & $8.0 \pm 7.0$ & 10.7 & 7.8 & 369 & 1.10 & 0.0024 & 0.090 & 4.32 & 0.75 \\
\hline$\overline{\mathrm{U} 1 \mathrm{c}}$ & FR & 78 & 18.9 & $0.625 \pm 0.37$ & 3.9 & 6.9 & 229 & 1.01 & 0.0058 & 0.171 & 3.10 & 0.55 \\
\hline $\mathrm{U} 2 \mathrm{c}$ & FR & 102 & 24.7 & $0.625 \pm 0.3$ & 5.1 & 7.3 & 272 & 1.16 & 0.0045 & 0.147 & 4.01 & 0.59 \\
\hline $\mathrm{J} 3 \mathrm{c}$ & FR & 126 & 30.5 & $0.625 \pm 0.375$ & 6.3 & 7.5 & 300 & 1.26 & 0.0038 & 0.129 & 4.85 & 0.61 \\
\hline J4c & FR & 156 & 37.8 & $0.625 \pm 0.3$ & 7.8 & 7.6 & 325 & 1. & 0.0032 & 0.113 & 5.99 & 0.62 \\
\hline $\mathrm{U} 5 \mathrm{c}$ & FR & 190 & 46.0 & $0.625 \pm 0.375$ & 9.5 & 7.7 & 364 & 1.56 & 0.0027 & 0.102 & 8.26 & 0.63 \\
\hline J6c & FR & 204 & 49.4 & $0.625 \pm 0.375$ & 10.2 & 7.6 & 366 & 1.57 & 0.0026 & 0.097 & 8.72 & 0.63 \\
\hline $\mathrm{U} 7 \mathrm{c}$ & FR & 228 & 55.2 & $0.625 \pm 0.375$ & 11.4 & 7.8 & 396 & 1.65 & 0.0023 & 0.092 & 9.89 & 0.64 \\
\hline$\overline{\mathrm{U} 8 \mathrm{c}}$ & $\overline{F R}$ & 18 & 20.8 & $3.0 \pm 2.0$ & 4.3 & 5.8 & 227 & 0.78 & 0.0063 & 0.186 & 1.82 & $\overline{0.47}$ \\
\hline J9c & FR & 23 & 26.6 & $3.0 \pm 2.0$ & 5.5 & 6.5 & 269 & 0. & 48 & 0.154 & 2.01 & 0.50 \\
\hline U10c & FR & 28 & 32.4 & \pm 2.0 & 6.7 & 6.9 & 304 & 0.95 & 039 & 0.134 & 2.03 & 0.53 \\
\hline U11c & FR & 35 & 40.2 & $3.0 \pm 2.0$ & 8.3 & 7.1 & 334 & 1.00 & 0.0033 & 0.117 & 2.26 & 0.54 \\
\hline $\mathrm{U} 12 \mathrm{c}$ & FR & 41 & 47.9 & $.0 \pm 2.0$ & 9.9 & 7.2 & 359 & 1.05 & 0.0028 & 0.103 & 2.46 & 0.57 \\
\hline $\mathrm{U} 13 \mathrm{c}$ & FR & 44 & 51.3 & \pm 2.0 & 10.6 & 7.4 & 383 & 1.09 & 0.0026 & 0.100 & 6.20 & 0.57 \\
\hline $\mathrm{U} 14 \mathrm{c}$ & FR & 50 & 58.1 & $3.0 \pm 2.0$ & 12.0 & 7.7 & 433 & 1.09 & 0.0024 & 0.096 & 6.11 & 0.58 \\
\hline U15c & FR & 7 & 20.3 & $8.0 \pm 7.0$ & 4.2 & 4.8 & 171 & 0.80 & 0.0068 & 0.174 & 2.08 & 0.50 \\
\hline U16c & FR & 8 & 25.7 & $8.0 \pm 7.0$ & 5.3 & 5.4 & 207 & 0.89 & 0.0052 & 0.147 & 1.77 & 0.51 \\
\hline U17c & FR & 10 & 32.4 & $8.0 \pm 7.0$ & 6.7 & 5.8 & 238 & 0.95 & 0.0042 & 0.126 & 2.63 & 0.53 \\
\hline $\mathrm{U} 18 \mathrm{c}$ & FR & 13 & 39.2 & $8.0 \pm 7.0$ & 8.1 & 6.2 & 272 & 1.00 & 0.0034 & 0.111 & 3.75 & 0.55 \\
\hline U19c & FR & 15 & 47.9 & $8.0 \pm 7.0$ & 9.9 & 6.5 & 298 & 1.01 & 0.0028 & 0.096 & 3.15 & 0.56 \\
\hline U20c & FR & 16 & 50.4 & $8.0 \pm 7.0$ & 10.4 & 6.4 & 299 & 1.05 & 0.0027 & 0.092 & 4.53 & 0.56 \\
\hline U21c & FR & 18 & 56.2 & $8.0 \pm 7.0$ & 11.6 & 6.9 & 348 & 1.05 & 0.0025 & 0.090 & 4.97 & 0.57 \\
\hline $\mathrm{E} 1$ & Open & - & 19.9 & - & 4.1 & 1.7 & 45 & 0.89 & 0.0101 & 0.134 & 1.64 & 0.11 \\
\hline E2 & Open & - & 29.5 & - & 6.1 & 1.6 & 50 & 1.00 & 0.0073 & 0.102 & 2.34 & 0.10 \\
\hline E3 & Open & - & 38.7 & - & 8.0 & 1.7 & 57 & 1.00 & 0.0058 & 0.086 & 3.00 & 0.09 \\
\hline E4 & Open & - & 49.9 & - & 10.3 & 1.7 & 65 & 1.01 & 0.0047 & 0.075 & 5.77 & 0.08 \\
\hline E5 & Open & - & 63.0 & - & 13.0 & 1.8 & 80 & 1.03 & 0.0040 & 0.070 & 7.82 & 0.08 \\
\hline E6 & Open & - & 67.8 & - & 14.0 & 1.8 & 80 & 1.06 & 0.0037 & 0.066 & 7.11 & 0.08 \\
\hline E7 & Open & - & 78.0 & - & 16.1 & 1.8 & 85 & 1.05 & 0.0034 & 0.061 & 8.10 & 0.08 \\
\hline
\end{tabular}




\begin{tabular}{|c|c|c|c|c|c|c|c|c|c|c|c|c|c|c|c|c|}
\hline Case & Mode & $R o$ & $\begin{array}{r}R e_{M} \\
\times 10^{3}\end{array}$ & $\begin{array}{c}T \pm t \\
{[\mathrm{~s}]}\end{array}$ & $\begin{array}{c}\Omega \pm \omega \\
{[\mathrm{Hz}]}\end{array}$ & $\begin{array}{c}A \pm \alpha \\
{[\mathrm{Hz} / \mathrm{s}]}\end{array}$ & $\begin{array}{c}U \\
{[\mathrm{~m} / \mathrm{s}]}\end{array}$ & $\begin{array}{c}T_{q} \\
{[\%]}\end{array}$ & $R e_{\lambda}$ & $\begin{array}{c}\langle\epsilon\rangle M / U^{3} \\
\times 10^{3}\end{array}$ & $u^{\prime} / v^{\prime}$ & $\eta / M$ & $\lambda / M$ & $L_{u x} / M$ & $L_{v x} / M$ & $L_{u y} / M$ \\
\hline$\overline{\mathrm{T} 1 \mathrm{a}}$ & FR & 17 & 38.7 & $2.11 \pm 2.07$ & $6.0 \pm 4.0$ & 125 & 8.0 & 6.8 & 241 & 0.23 & 1.15 & 0.0028 & 0.085 & 1.41 & 0.98 & \\
\hline $\mathrm{T} 2 \mathrm{a}$ & FR & 17 & 38.7 & $2.12 \pm 2.07$ & $6.0 \pm 4.0$ & 125 & 8.0 & 6.6 & 226 & 0.23 & 1.19 & 0.0028 & 0.082 & 1.41 & 0.75 & \\
\hline T3a & FR & 17 & 38.7 & $2.48 \pm 2.44$ & $6.0 \pm 4.0$ & 125 & 8.0 & 6.6 & 227 & 0.23 & 1.15 & 0.0028 & 0.083 & 1.33 & 0.83 & \\
\hline $\mathrm{T} 4 \mathrm{a}$ & FR & 17 & 38.7 & $3.18 \pm 3.14$ & $6.0 \pm 4.0$ & 125 & 8.0 & 6.7 & 235 & 0.22 & 1.17 & 0.0028 & 0.084 & 1.42 & 0.83 & \\
\hline T5a & FR & 17 & 39.2 & $5.40 \pm 5.36$ & $6.0 \pm 4.0$ & 125 & 8.1 & 6.6 & 236 & 0.22 & 1.14 & 0.0028 & 0.085 & 1.31 & 0.85 & \\
\hline T6a & FR & 17 & 38.7 & $7.78 \pm 2.69$ & $6.0 \pm 4.0$ & 125 & 8.0 & 6.7 & 237 & 0.22 & 1.13 & 0.0028 & 0.086 & 1.28 & 0.86 & \\
\hline V1a & FR & 166 & 40.2 & $2.11 \pm 2.02$ & $0.625 \pm 0.375$ & 125 & 8.3 & 7.4 & 323 & 0.18 & 1.75 & 0.0028 & 0.092 & 7.16 & 2.07 & \\
\hline $\mathrm{V} 2 \mathrm{a}$ & FR & 81 & 39.2 & $2.11 \pm 2.06$ & $1.25 \pm 0.75$ & 125 & 8.1 & 7.5 & 312 & 0.20 & 1.50 & 0.0027 & 0.089 & 3.29 & 1.61 & 1.18 \\
\hline V3a & FR & 50 & 38.7 & $2.11 \pm 2.06$ & $2.0 \pm 1.0$ & 125 & 8.0 & 7.4 & 312 & 0.20 & 1.36 & 0.0027 & 0.090 & 2.27 & 1.27 & \\
\hline $\mathrm{V} 4 \mathrm{a}$ & FR & 35 & 40.2 & $2.12 \pm 2.04$ & $3.0 \pm 2.0$ & 250 & 8.3 & 7.2 & 271 & 0.24 & 1.29 & 0.0028 & 0.089 & 2.13 & 1.08 & 0.98 \\
\hline V5a & FR & 19 & 40.2 & $2.17 \pm 2.01$ & $5.5 \pm 4.5$ & 250 & 8.3 & 6.6 & 237 & 0.21 & 1.25 & 0.0028 & 0.086 & 1.58 & 0.80 & 0.91 \\
\hline V6a & FR & 13 & 39.2 & $2.11 \pm 2.06$ & $8.0 \pm 7.0$ & 125 & 8.1 & 6.3 & 219 & 0.20 & 1.22 & 0.0029 & 0.085 & 1.63 & 0.74 & 0.86 \\
\hline V7a & FR & 9 & 37.3 & $2.11 \pm 2.02$ & $10.5 \pm 9.5$ & 125 & 7.7 & 6.3 & 199 & 0.23 & 1.22 & 0.0029 & 0.081 & 1.41 & 0.71 & \\
\hline V8a & FR & 8 & 36.8 & $2.11 \pm 2.05$ & $11.5 \pm 8.5$ & 125 & 7.6 & 6.0 & 185 & 0.22 & 1.19 & 0.0030 & 0.079 & 1.21 & 0.65 & \\
\hline V9a & FR & 8 & 37.3 & $2.11 \pm 2.03$ & $12.5 \pm 7.5$ & 125 & 7.7 & 6.0 & 178 & 0.23 & 1.17 & 0.0029 & 0.076 & 2.51 & 0.58 & \\
\hline V10a & FR & 7 & 38.3 & $2.12 \pm 2.07$ & $15.0 \pm 5.0$ & 125 & 7.9 & 5.3 & 162 & 0.19 & 1.13 & 0.0030 & 0.075 & 1.42 & 0.60 & \\
\hline V11a & FR & 6 & 38.3 & $2.11 \pm 2.04$ & $17.5 \pm 2.5$ & 125 & 7.9 & 5.2 & 177 & 0.14 & 1.15 & 0.0031 & 0.076 & 2.79 & 0.65 & \\
\hline$\overline{\mathrm{A} 1 \mathrm{a}}$ & FR & 14 & 42.6 & $2.12 \pm 2.03$ & $8.0 \pm 7.0$ & 25 & 8.8 & 6.5 & 218 & 0.25 & 1.18 & 0.0026 & 0.075 & 2.52 & 0.80 & \\
\hline $\mathrm{A} 2 \mathrm{a}$ & FR & 15 & 45.0 & $2.12 \pm 2.04$ & $8.0 \pm 7.0$ & $0.625 \pm 0.375$ & 9.3 & 6.4 & 234 & 0.21 & 1.18 & 0.0026 & 0.078 & 1.54 & 0.81 & \\
\hline A3a & FR & 13 & 41.6 & $2.12 \pm 2.07$ & $8.0 \pm 7.0$ & $50 \pm 25$ & 8.6 & 6.6 & 219 & 0.27 & 1.21 & 0.0026 & 0.075 & 3.09 & 0.80 & \\
\hline $\mathrm{A} 4 \mathrm{a}$ & FR & 13 & 41.6 & $2.12 \pm 2.03$ & $8.0 \pm 7.0$ & $62.5 \pm 37.5$ & 8.6 & 6.6 & 213 & 0.28 & 1.17 & 0.0026 & 0.074 & 1.64 & 0.80 & \\
\hline $\mathrm{A} 5 \mathrm{a}$ & FR & 14 & 44.6 & $2.11 \pm 2.07$ & $8.0 \pm 7.0$ & $137.5 \pm 12.5$ & 9.2 & 6.4 & 231 & 0.23 & 1.18 & 0.0026 & 0.077 & 1.59 & 0.86 & \\
\hline A6a & $\mathrm{CL}$ & 26 & 40.7 & $2.49 \pm 2.45$ & $4.0 \pm 2.0$ & 5 & 8.4 & 6.8 & 262 & 0.19 & 1.23 & 0.0028 & 0.091 & 1.55 & 0.93 & \\
\hline $\mathrm{A} 7 \mathrm{a}$ & $\mathrm{CL}$ & 25 & 39.2 & $2.48 \pm 2.44$ & $4.0 \pm 2.0$ & 10 & 8.1 & 7.0 & 249 & 0.24 & 1.21 & 0.0028 & 0.087 & 1.51 & 0.94 & \\
\hline A8a & CL & 25 & 39.2 & $2.48 \pm 2.44$ & $4.0 \pm 2.0$ & 20 & 8.1 & 7.1 & 253 & 0.24 & 1.18 & 0.0028 & 0.088 & 1.50 & 1.00 & \\
\hline$\overline{\text { S1a }}$ & $\mathrm{CL}$ & 13 & 41.2 & $2.11 \pm 2.07$ & $8.0 \pm 7.0$ & 200 & 8.5 & 7.1 & 270 & 0.23 & 1.22 & 0.0025 & 0.077 & 3.27 & 0.86 & \\
\hline S2a & CL & 12 & 37.3 & $2.11 \pm 2.07$ & $8.0 \pm 7.0$ & 200 & 7.7 & 6.2 & 218 & 0.18 & 1.19 & 0.0031 & 0.090 & 1.46 & 0.77 & \\
\hline S3a & $\mathrm{CL}$ & 9 & 26.6 & $2.11 \pm 2.07$ & $8.0 \pm 7.0$ & 200 & 5.5 & 5.6 & 172 & 0.14 & 1.20 & 0.0042 & 0.110 & 1.31 & 0.68 & \\
\hline $\mathrm{S} 4 \mathrm{a}$ & $\mathrm{CL}$ & 154 & 37.3 & $2.11 \pm 2.07$ & $0.625 \pm 0.375$ & 200 & 7.7 & 7.6 & 280 & 0.25 & 1.72 & 0.0027 & 0.083 & 6.36 & 1.94 & \\
\hline S5a & $\mathrm{CL}$ & 51 & 39.2 & $2.10 \pm 2.06$ & $2.0 \pm 1.0$ & 200 & 8.1 & 7.5 & 280 & 0.25 & 1.29 & 0.0027 & 0.090 & 2.30 & 1.56 & \\
\hline S6a & $\mathrm{CL}$ & 35 & 40.2 & $2.11 \pm 2.07$ & $3.0 \pm 2.0$ & 250 & 8.3 & 7.2 & 268 & 0.23 & 1.28 & 0.0028 & 0.089 & 1.88 & 1.08 & 1.00 \\
\hline S7a & $\mathrm{CL}$ & 17 & 40.2 & $2.10 \pm 2.06$ & $6.0 \pm 4.0$ & 200 & 8.3 & 6.6 & 237 & 0.21 & 1.16 & 0.0028 & 0.085 & 1.39 & 0.83 & \\
\hline$\overline{\mathrm{C} 1 \mathrm{a}}$ & $10 \mathrm{CL}$ & 12 & 38.3 & $2.12 \pm 2.08$ & $8.0 \pm 7.0$ & 200 & 7.9 & 6.5 & 216 & 1.57 & 1.18 & 0.0028 & 0.082 & 2.56 & 0.79 & \\
\hline $\mathrm{C} 2 \mathrm{a}$ & $5 \mathrm{FR}$ & 12 & 38.3 & $2.11 \pm 2.07$ & $8.0 \pm 7.0$ & 200 & 7.9 & 6.4 & 215 & 0.99 & 1.18 & 0.0029 & 0.082 & 1.63 & 0.78 & \\
\hline
\end{tabular}


Table A2 - Continued

\begin{tabular}{|c|c|c|c|c|c|c|c|c|c|c|c|c|c|c|c|c|}
\hline Case & Mode & $R o$ & $\begin{array}{l}R_{M} \\
\times 10^{3}\end{array}$ & $\begin{array}{c}T \pm t \\
{[\mathrm{~s}]}\end{array}$ & $\begin{array}{c}\Omega \pm \omega \\
{[\mathrm{Hz}]}\end{array}$ & $\begin{array}{l}A \pm \alpha \\
{[\mathrm{Hz} / \mathrm{s}]}\end{array}$ & $\begin{array}{c}U \\
{[\mathrm{~m} / \mathrm{s}]}\end{array}$ & $\begin{array}{c}T_{q} \\
{[\%]}\end{array}$ & $R e_{\lambda}$ & $\begin{array}{c}\langle\epsilon\rangle M / U^{3} \\
\quad \times 10^{3}\end{array}$ & $u^{\prime} / v^{\prime}$ & $\eta / M$ & $\lambda / M$ & $L_{u x} / M$ & $L_{v x} / M$ & $L_{u y} / M$ \\
\hline$\overline{\mathrm{C} 3 \mathrm{a}}$ & $6 \mathrm{CL}$ & 13 & 38.7 & $2.14 \pm 2.10$ & $8.0 \pm 7.0$ & 200 & 8.0 & 6.5 & 223 & 0.93 & 1.19 & 0.0028 & 0.083 & 1.62 & 0.79 & \\
\hline $\mathrm{C} 4 \mathrm{a}$ & 3FR & 13 & 38.7 & $2.14 \pm 2.10$ & $8.0 \pm 7.0$ & 200 & 8.0 & 6.5 & 221 & 0.86 & 1.16 & 0.0028 & 0.083 & 1.43 & 0.87 & \\
\hline C5a & $4 C L$ & 13 & 38.7 & $2.14 \pm 2.10$ & $8.0 \pm 7.0$ & 200 & 8.0 & 6.5 & 216 & 1.56 & 1.20 & 0.0028 & 0.082 & 2.68 & 0.75 & \\
\hline C6a & $2 \mathrm{FR}$ & 13 & 38.7 & $2.14 \pm 2.10$ & $8.0 \pm 7.0$ & 200 & 8.0 & 6.6 & 229 & 1.86 & 1.22 & 0.0028 & 0.085 & 3.46 & 0.82 & \\
\hline $\mathrm{C} 7 \mathrm{a}$ & $10 \mathrm{CL}$ & 51 & 39.2 & $2.12 \pm 2.08$ & $2.0 \pm 1.0$ & 200 & 8.1 & 7.7 & 321 & 0.82 & 1.33 & 0.0027 & 0.090 & 2.34 & 1.46 & \\
\hline $\mathrm{C} 8 \mathrm{a}$ & $5 F R$ & 51 & 39.2 & $2.12 \pm 2.07$ & $2.0 \pm 1.0$ & 200 & 8.1 & 7.6 & 280 & 0.83 & 1.35 & 0.0027 & 0.089 & 2.38 & 1.33 & \\
\hline $\mathrm{C} 9 \mathrm{a}$ & $6 \mathrm{CL}$ & 51 & 39.2 & $2.16 \pm 2.12$ & $2.0 \pm 1.0$ & 200 & 8.1 & 7.5 & 274 & 0.81 & 1.39 & 0.0027 & 0.088 & 2.32 & 1.20 & \\
\hline $\mathrm{C} 10 \mathrm{a}$ & $3 F R$ & 51 & 39.2 & $2.15 \pm 2.11$ & $2.0 \pm 1.0$ & 200 & 8.1 & 7.6 & 281 & 0.84 & 1.33 & 0.0027 & 0.089 & 2.39 & 1.39 & \\
\hline C11a & $4 C L$ & 51 & 39.2 & $2.16 \pm 2.11$ & $2.0 \pm 1.0$ & 200 & 8.1 & 7.5 & 270 & 0.83 & 1.31 & 0.0027 & 0.088 & 2.17 & 1.30 & \\
\hline $\mathrm{C} 12 \mathrm{a}$ & $2 \mathrm{FR}$ & 51 & 39.7 & $2.14 \pm 2.09$ & $2.0 \pm 1.0$ & 200 & 8.2 & 7.5 & 285 & 0.80 & 1.35 & 0.0027 & 0.091 & 2.36 & 1.41 & \\
\hline $\mathrm{C} 13 \mathrm{a}$ & $10 \mathrm{CL}$ & 33 & 38.3 & $2.12 \pm 2.08$ & $3.0 \pm 2.0$ & 250 & 7.9 & 7.2 & 250 & 0.76 & 1.17 & 0.0028 & 0.086 & 1.55 & 1.28 & 0.99 \\
\hline$\overline{\text { G1a }}$ & $\overline{F R}$ & 12 & 38.3 & $2.12 \pm 2.02$ & $8.0 \pm 7.0$ & 200 & 7.9 & 6.4 & 214 & 0.22 & 1.17 & 0.0029 & 0.082 & 2.15 & 0.76 & \\
\hline $\mathrm{G} 2 \mathrm{a}$ & FR & 50 & 38.7 & $2.12 \pm 2.03$ & $2.0 \pm 1.0$ & 200 & 8.0 & 7.5 & 300 & 0.21 & 1.36 & 0.0027 & 0.088 & 2.19 & 1.27 & \\
\hline G3a & CL & 13 & 39.2 & $2.11 \pm 2.07$ & $8.0 \pm 7.0$ & 200 & 8.1 & 6.6 & 237 & 0.22 & 1.21 & 0.0028 & 0.086 & 3.59 & 0.73 & \\
\hline G4a & $\mathrm{CL}$ & 50 & 38.7 & $2.11 \pm 2.06$ & $2.0 \pm 1.0$ & 200 & 8.0 & 7.4 & 267 & 0.26 & 1.33 & 0.0027 & 0.088 & 2.11 & 1.35 & \\
\hline$\overline{\mathrm{R} 1 \mathrm{a}}$ & FR & 13 & 41.2 & $2.11 \pm 2.07$ & $8.0 \pm 7.0$ & 250 & 8.5 & 6.3 & 250 & 0.16 & 1.26 & 0.0030 & 0.092 & 1.88 & 0.89 & \\
\hline $\mathrm{R} 2 \mathrm{a}$ & FR & 53 & 41.2 & $2.12 \pm 2.07$ & $2.0 \pm 1.0$ & 250 & 8.5 & 7.1 & 290 & 0.19 & 1.38 & 0.0028 & 0.095 & 2.47 & 1.34 & \\
\hline R3a & CL & 53 & 40.7 & $2.10 \pm 2.06$ & $2.0 \pm 1.0$ & 250 & 8.4 & 7.2 & 329 & 0.16 & 1.38 & 0.0028 & 0.096 & 2.49 & 1.46 & \\
\hline
\end{tabular}




\begin{tabular}{|c|c|c|c|c|c|c|c|c|c|c|c|c|c|c|c|c|}
\hline$\overline{\text { Case }}$ & Mode & $R o$ & $\begin{array}{l}R_{M} \\
\times 10^{3} \\
\end{array}$ & $\begin{array}{c}T \pm t \\
{[\mathrm{~s}]}\end{array}$ & $\begin{array}{c}\Omega \pm \omega \\
{[\mathrm{Hz}]}\end{array}$ & $\begin{array}{c}A \pm \alpha \\
{[\mathrm{Hz} / \mathrm{s}]}\end{array}$ & $\begin{array}{c}U \\
{[\mathrm{~m} / \mathrm{s}]}\end{array}$ & $\begin{array}{c}T_{q} \\
{[\%]}\end{array}$ & $R e_{\lambda}$ & $\begin{array}{c}\langle\epsilon\rangle M / U^{3} \\
\times 10^{3}\end{array}$ & $u^{\prime} / v^{\prime}$ & $\eta / M$ & $\lambda / M$ & $L_{u x} / M$ & $L_{v x} / M$ & $\overline{L u y} / M$ \\
\hline$\overline{\mathrm{T} 1 \mathrm{~b}}$ & FR & 16 & 38.3 & $2.11 \pm 2.07$ & $6.0 \pm 4.0$ & 125 & 7.9 & 8.6 & 285 & 0.42 & 1.19 & 0.0024 & 0.079 & 1.58 & 0.91 & \\
\hline $\mathrm{T} 4 \mathrm{~b}$ & FR & 18 & 40.7 & $3.18 \pm 3.14$ & $6.0 \pm 4.0$ & 125 & 8.4 & 8.2 & 302 & 0.33 & 1.17 & 0.0024 & 0.083 & 1.62 & 0.94 & \\
\hline T5b & FR & 17 & 38.7 & $5.40 \pm 5.36$ & $6.0 \pm 4.0$ & 125 & 8.0 & 8.4 & 283 & 0.39 & 1.15 & 0.0024 & 0.080 & 1.45 & 0.90 & \\
\hline T6b & FR & 17 & 38.7 & $7.78 \pm 2.69$ & $6.0 \pm 4.0$ & 125 & 8.0 & 8.4 & 286 & 0.38 & 1.13 & 0.0024 & 0.081 & 1.38 & 0.95 & \\
\hline $\mathrm{V} 1 \mathrm{~b}$ & FR & 160 & 38.7 & $2.11 \pm 2.02$ & $0.625 \pm 0.375$ & 125 & 8.0 & 9.6 & 426 & 0.30 & 1.64 & 0.0024 & 0.092 & 6.19 & 2.92 & \\
\hline $\mathrm{V} 2 \mathrm{~b}$ & FR & 80 & 38.7 & $2.11 \pm 2.06$ & $1.25 \pm 0.75$ & 125 & 8.0 & 9.6 & 423 & 0.30 & 1.41 & 0.0024 & 0.091 & 3.30 & 2.03 & \\
\hline V3b & FR & 51 & 39.7 & $2.11 \pm 2.06$ & $2.0 \pm 1.0$ & 125 & 8.2 & 9.5 & 376 & 0.37 & 1.24 & 0.0024 & 0.092 & 2.26 & 1.46 & \\
\hline $\mathrm{V} 4 \mathrm{~b}$ & FR & 33 & 38.3 & $2.11 \pm 2.07$ & $3.0 \pm 2.0$ & 125 & 7.9 & 9.6 & 353 & 0.42 & 1.24 & 0.0024 & 0.089 & 3.67 & 1.31 & 1.00 \\
\hline V5b & FR & 17 & 38.7 & $2.11 \pm 2.03$ & $5.5 \pm 4.5$ & 125 & 8.0 & 8.6 & 303 & 0.38 & 1.21 & 0.0025 & 0.084 & 1.81 & 1.08 & 0.93 \\
\hline V6b & FR & 12 & 38.3 & $2.11 \pm 2.06$ & $8.0 \pm 7.0$ & 125 & 7.9 & 8.0 & 274 & 0.33 & 1.23 & 0.0025 & 0.083 & 2.52 & 0.96 & 0.89 \\
\hline $\mathrm{V} 7 \mathrm{~b}$ & FR & 10 & 38.7 & $2.11 \pm 2.02$ & $10.5 \pm 9.5$ & 125 & 8.0 & 7.7 & 269 & 0.30 & 1.22 & 0.0026 & 0.084 & 1.70 & 0.90 & \\
\hline V8b & FR & 9 & 38.3 & $2.11 \pm 2.05$ & $11.5 \pm 8.5$ & 125 & 7.9 & 7.4 & 250 & 0.30 & 1.20 & 0.0026 & 0.082 & 2.69 & 0.85 & \\
\hline V10b & FR & 7 & 38.3 & $2.12 \pm 2.07$ & $15.0 \pm 5.0$ & 125 & 7.9 & 6.6 & 222 & 0.24 & 1.20 & 0.0027 & 0.081 & 1.68 & 0.84 & \\
\hline V11b & FR & 6 & 39.2 & $2.11 \pm 2.04$ & $17.5 \pm 2.5$ & 125 & 8.1 & 6.3 & 227 & 0.20 & 1.22 & 0.0028 & 0.084 & 1.97 & 0.86 & \\
\hline$\overline{\mathrm{A} 2 \mathrm{~b}}$ & FR & 13 & 39.2 & $2.12 \pm 2.04$ & $8.0 \pm 7.0$ & $0.625 \pm 0.375$ & 8.1 & 7.7 & 276 & 0.30 & 1.26 & 0.0026 & 0.085 & 2.60 & 0.94 & \\
\hline $\mathrm{A} 5 \mathrm{~b}$ & FR & 13 & 39.2 & $2.11 \pm 2.07$ & $8.0 \pm 7.0$ & $137.5 \pm 12.5$ & 8.1 & 7.7 & 271 & 0.30 & 1.22 & 0.0026 & 0.083 & 1.70 & 0.96 & \\
\hline A6b & CL & 25 & 38.7 & $2.49 \pm 2.45$ & $4.0 \pm 2.0$ & 5 & 8.0 & 8.6 & 307 & 0.37 & 1.21 & 0.0025 & 0.085 & 1.63 & 1.04 & \\
\hline $\mathrm{A} 7 \mathrm{~b}$ & $\mathrm{CL}$ & 25 & 39.2 & $2.48 \pm 2.44$ & $4.0 \pm 2.0$ & 10 & 8.1 & 8.7 & 316 & 0.37 & 1.21 & 0.0024 & 0.086 & 1.68 & 1.05 & \\
\hline $\mathrm{A} 8 \mathrm{~b}$ & $\mathrm{CL}$ & 25 & 39.2 & $2.48 \pm 2.44$ & $4.0 \pm 2.0$ & 20 & 8.1 & 8.8 & 326 & 0.36 & 1.19 & 0.0025 & 0.087 & 1.62 & 1.15 & \\
\hline$\overline{\mathrm{S} 5 \mathrm{~b}}$ & $\mathrm{CL}$ & 51 & 39.7 & $2.10 \pm 2.06$ & $2.0 \pm 1.0$ & 200 & 8.2 & 9.3 & 357 & 0.38 & 1.30 & 0.0024 & 0.089 & 2.38 & 1.48 & \\
\hline S7b & CL & 17 & 39.2 & $2.10 \pm 2.06$ & $6.0 \pm 4.0$ & 200 & 8.1 & 8.3 & 297 & 0.36 & 1.19 & 0.0024 & 0.082 & 1.67 & 1.14 & \\
\hline$\overline{\mathrm{C} 1 \mathrm{~b}}$ & 10CL & 13 & 38.7 & $2.12 \pm 2.08$ & $8.0 \pm 7.0$ & 200 & 8.0 & 8.2 & 285 & 1.51 & 1.24 & 0.0025 & 0.083 & 3.67 & 0.85 & \\
\hline $\mathrm{C} 3 \mathrm{~b}$ & $6 \mathrm{CL}$ & 12 & 37.3 & $2.14 \pm 2.10$ & $8.0 \pm 7.0$ & 200 & 7.7 & 8.7 & 332 & 1.66 & 1.27 & 0.0024 & 0.082 & 3.91 & 0.92 & \\
\hline $\mathrm{C} 5 \mathrm{~b}$ & $4 \mathrm{CL}$ & 13 & 38.7 & $2.14 \pm 2.10$ & $8.0 \pm 7.0$ & 200 & 8.0 & 8.3 & 294 & 1.21 & 1.21 & 0.0025 & 0.085 & 2.90 & 1.03 & \\
\hline
\end{tabular}

Table A4: Grid and flow properties for test cases with solid circular wings. All measurements were performed at $x / M=30$.

\begin{tabular}{lcccccccccccccccc}
\hline Case & Mode & $R o$ & $\begin{array}{c}R e_{M} \\
\times 10^{3}\end{array}$ & $\begin{array}{c}T \pm t \\
{[\mathrm{~s}]}\end{array}$ & $\begin{array}{c}\Omega \pm \omega \\
{[\mathrm{Hz}]}\end{array}$ & $\begin{array}{c}A \pm \alpha \\
{[\mathrm{Hz} / \mathrm{s}]}\end{array}$ & $\begin{array}{c}U \\
{[\mathrm{~m} / \mathrm{s}]}\end{array}$ & $\begin{array}{c}T_{q} \\
{[\%]}\end{array}$ & $\begin{array}{c}R e_{\lambda} \\
\langle\epsilon\rangle M / U^{3} \\
\times 10^{3}\end{array}$ & $u^{\prime} / v^{\prime}$ & $\eta / M$ & $\lambda / M$ & $L_{u x} / M$ & $L_{v x} / M$ & $L_{u y} / M$ \\
\hline \hline V1c & FR & 168 & 40.7 & $2.11 \pm 2.02$ & $0.625 \pm 0.375$ & 125 & 8.4 & 8.0 & 379 & 1.19 & 1.67 & 0.0027 & 0.096 & 6.88 & 2.51 & \\
V2c & FR & 95 & 46.0 & $2.11 \pm 2.06$ & $1.25 \pm 0.75$ & 125 & 9.5 & 8.1 & 406 & 0.19 & 1.48 & 0.0024 & 0.089 & 3.80 & 1.76 & \\
V4c & FR & 35 & 40.2 & $2.12 \pm 2.04$ & $3.0 \pm 2.0$ & 250 & 8.3 & 7.9 & 318 & 0.25 & 1.23 & 0.0027 & 0.093 & 2.06 & 1.25 & 1.03 \\
V6c & FR & 13 & 40.2 & $2.12 \pm 2.07$ & $8.0 \pm 7.0$ & 250 & 8.3 & 6.9 & 258 & 0.22 & 1.24 & 0.0028 & 0.088 & 2.63 & 0.82 & \\
\hline S1c & CL & 13 & 40.7 & $2.11 \pm 2.07$ & $8.0 \pm 7.0$ & 200 & 8.4 & 6.9 & 262 & 0.21 & 1.19 & 0.0028 & 0.089 & 1.72 & 0.90 & \\
\hline C1c & 10CL & 13 & 40.7 & $2.12 \pm 2.08$ & $8.0 \pm 7.0$ & 200 & 8.4 & 6.8 & 260 & 1.03 & 1.13 & 0.0028 & 0.088 & 2.15 & 3.46 \\
\hline
\end{tabular}

